\title{
MASH Explorer: A Universal Software Environment for Top-Down Proteomics \\ User Manual
}

Version 2.0

Ying Ge Research Group - University of Wisconsin, Madison

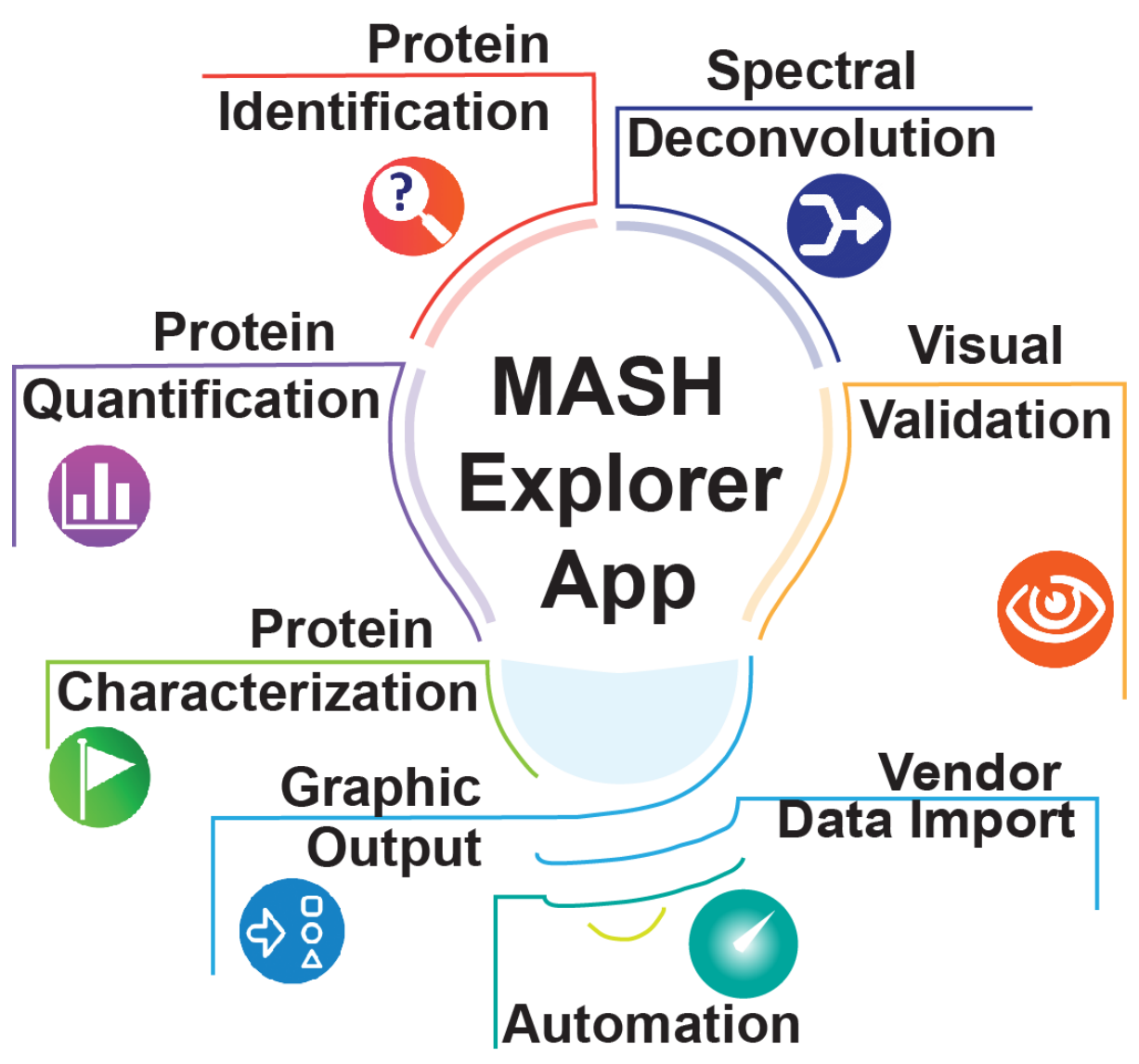

Copyright (C) 2020 Ying Ge Research Group, University of Wisconsin School of Medicine and Public Health 


\section{Table of Contents}

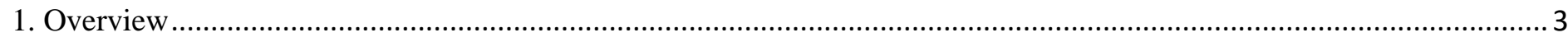

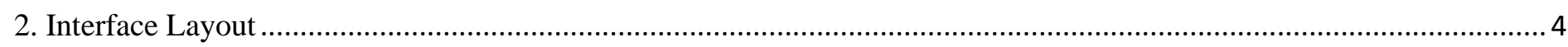

3. Discovery Mode: Protein Identification and Characterization from LC-MS/MS Data ............................................. 5

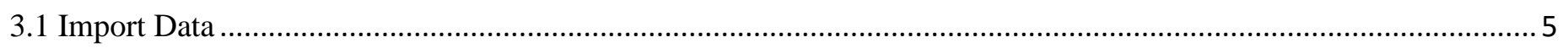

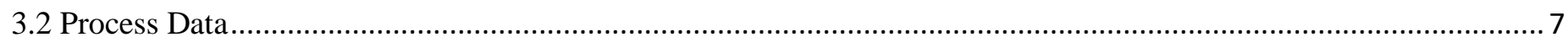

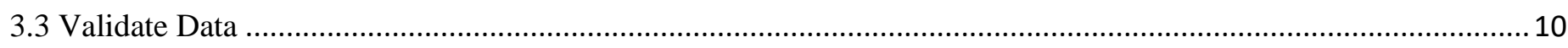

4. Targeted Mode: Protein Identification and Characterization from MS/MS Data ................................................... 12

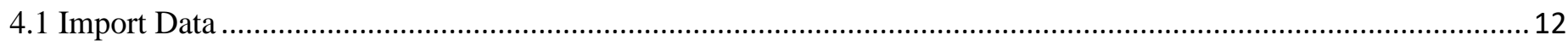

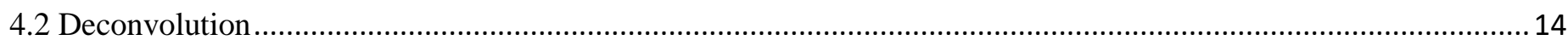

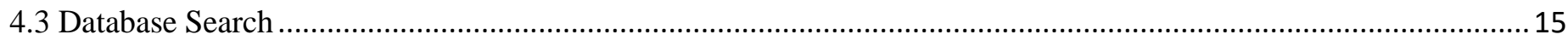

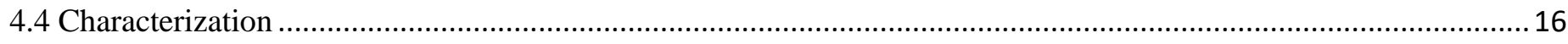

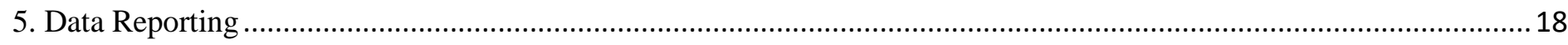

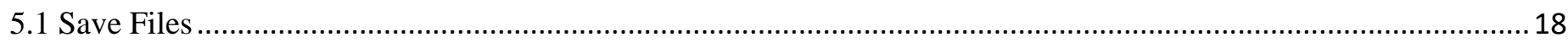

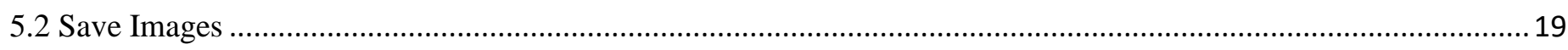

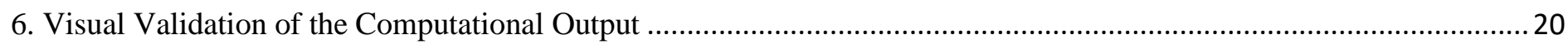

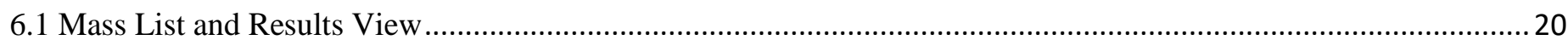

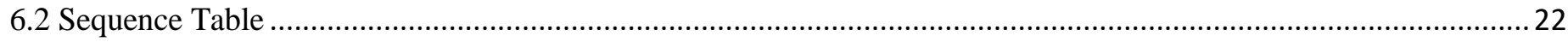

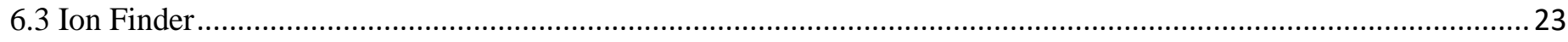

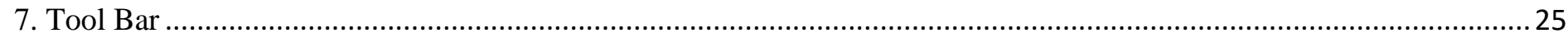

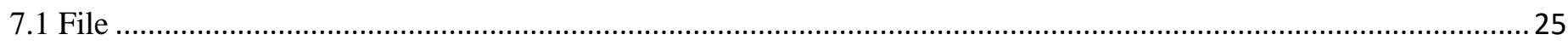

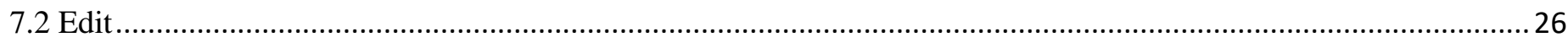

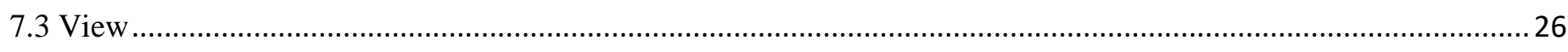

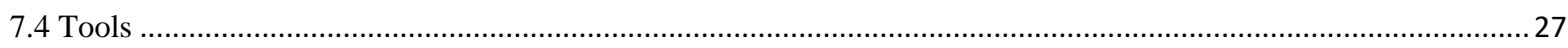

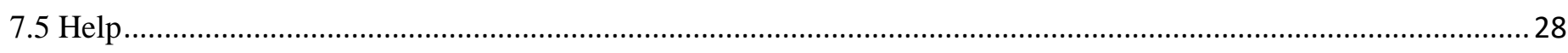

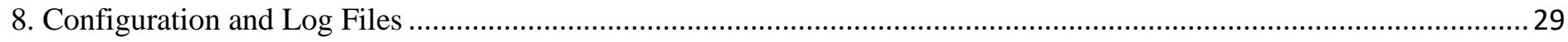

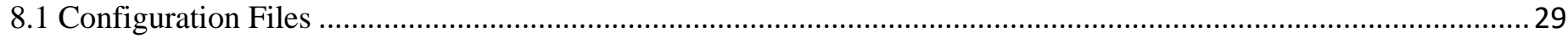

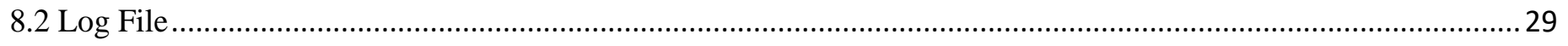




\section{Overview}

MASH Explorer, developed by the Ying Ge research group, is a comprehensive, universal, user-friendly, and free software environment for top-down proteomics data processing. MASH Explorer allows data import from various vendor data formats and accommodates several deconvolution and database search pipelines for spectral deconvolution and protein identification. MASH Explorer provides visualization tools for complex top-down mass spectra to validate deconvolution and protein identification results.

This tutorial provides step-by-step instructions for protein identification in Discovery mode (large-scale protein identification), Targeted mode (comprehensive protein characterization), and visual validation of results.

The development of MASH Explorer is supported by National Institute of Health R01 GM125085. 


\section{Interface Layout}

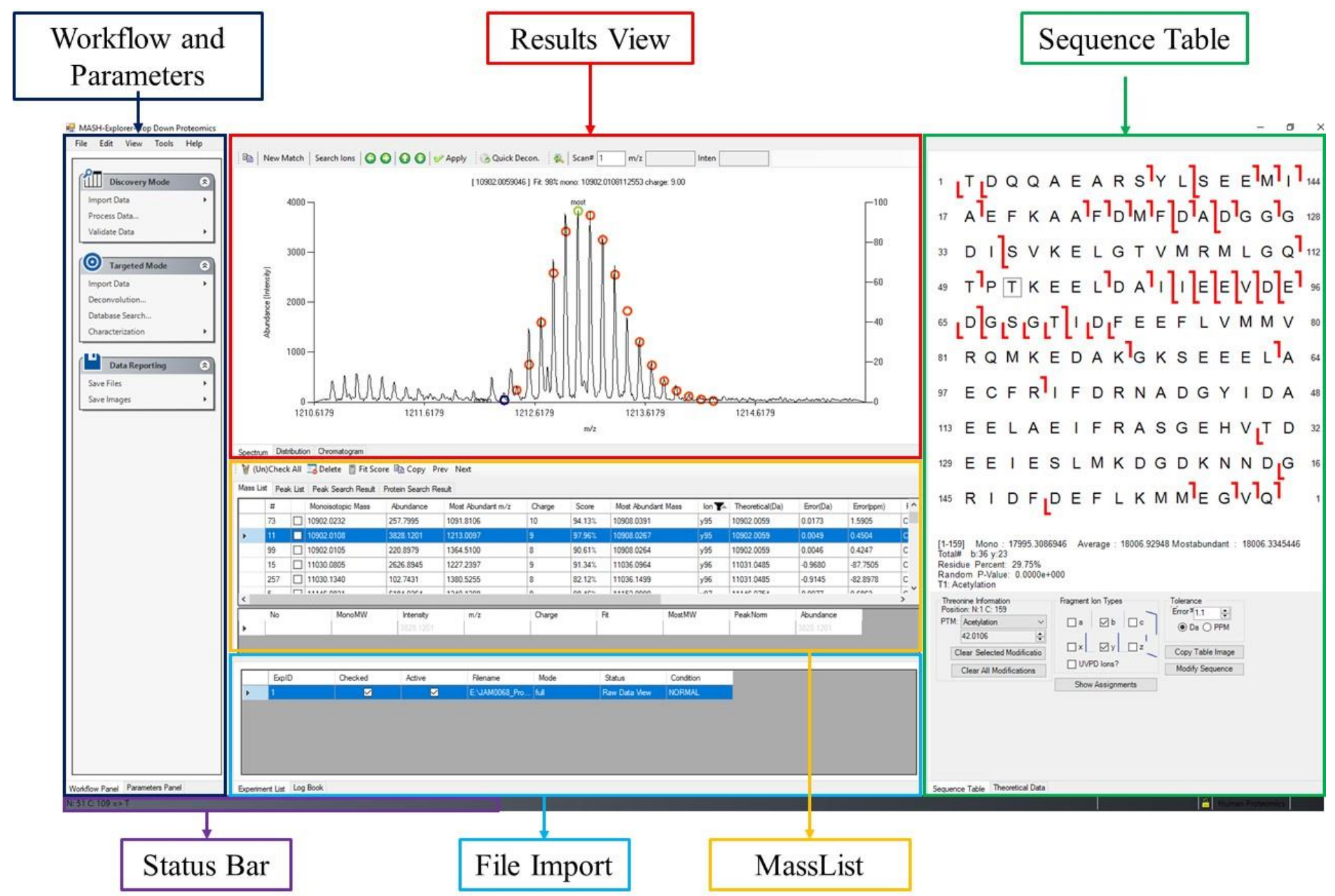

Figure 2.1 Overview of MASH Explorer software Interface

Workflow and Parameters: Users can follow the steps for LC-MS/MS and MS/MS workflow for data processing.

Results View: The mass spectra and chromatogram are shown in this panel.

Mass List: The fragment ion information is presented in this panel.

File Import: This panel allows for multiple files to be processed at the same time.

LogBook: This panel displays processing and error messages.

Note: File Import and LogBook are tabs in the same panel of the MASH Explorer App window.

Status Bar: The progress of data processing is shown in this panel.

Sequence Table: The protein sequence and top-down fragment ion information is presented in this panel.

Note: The V2.0 interface changed from the docking panels of previous versions to using split views. 


\section{Discovery Mode: Protein Identification and Characterization from LC-MS/MS Data}

Our team designed the Discovery Mode workflow to handle batch LC-MS/MS analysis. This workflow includes data import, data processing including deconvolution and database search, and finally data validation for protein identification.

\begin{tabular}{|c|c|}
\hline Discovery Mode & 스 \\
\hline Import Data & • \\
\hline Process Data... & \\
\hline Validate Data & $\bullet$ \\
\hline
\end{tabular}

Figure 3.0.1 Discovery Mode button layout

\subsection{Import Data}

In the Discovery Mode tab under the Workflow and Parameters panel, "Import Data" will direct users to load experimental data to MASH Explorer. Users can follow the steps below for data import:

1. Click on "Import Data" in the Discovery Mode tab under the Workflow and Parameters panel to add a data set (Figure 3.1.1). *

2. Click on the option that represents the raw data file format that will be uploaded (Figure 3.1.1). The current version of MASH Explorer supports Thermo (.raw), Bruker (.baf), Bruker data output in ASCII format (.ascii), Waters (.raw), and universal file formats (mzXML and MGF).

3. Select the LC-MS/MS data files to be analyzed. The Status Bar will update the data importing progress (Figure 3.1.2).

4. The current version of MASH Explorer allows users to have multiple files open at the same time. Users can sequentially open data files.
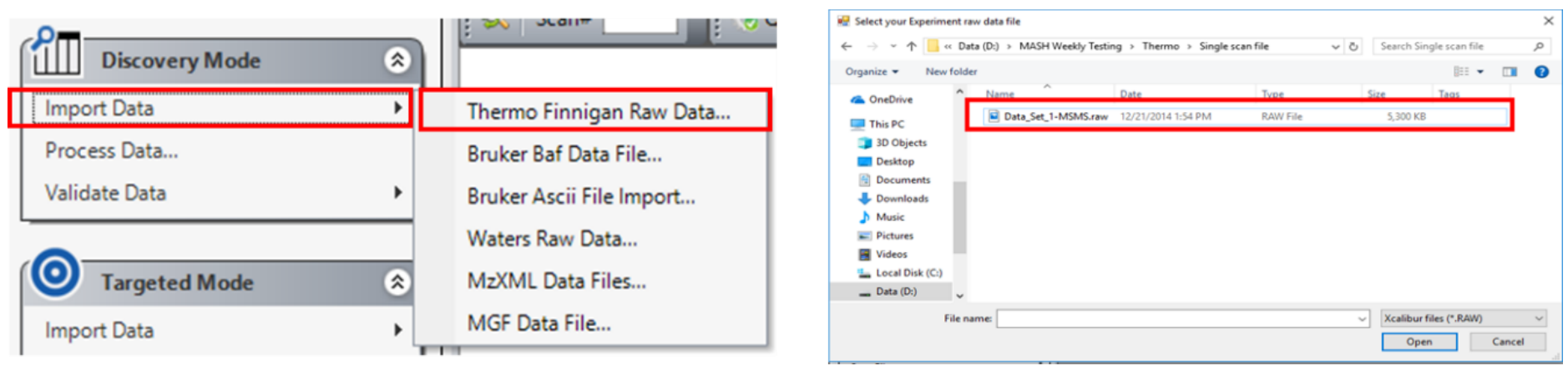

Figure 3.1.1 Import Data function and file selection 


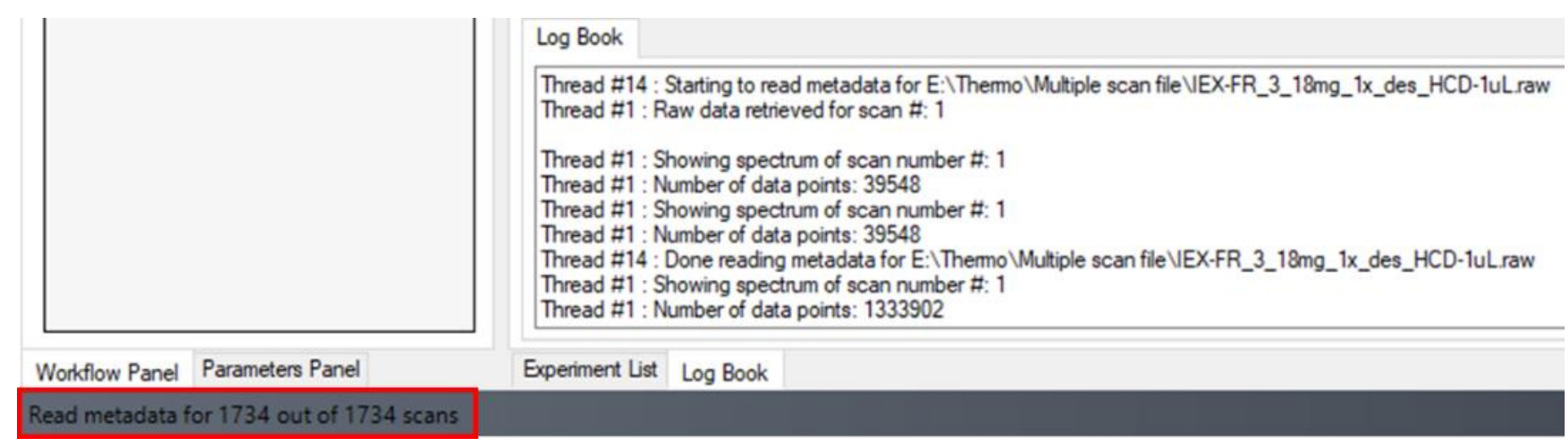

Figure 3.1.2 Status Bar panel updates for data import

* The user can also choose to drag and drop the data file to import data into the software.

Users can view the chromatogram of the imported data and navigate different scans. The chromatogram can be visualized in the "Chromatogram" tab in the Results View panel (Figure 3.1.3).

Users can navigate to different scans in three ways:

- By scrolling the scan list and selecting the desired scan or scans.

- By double-clicking in the chromatogram view - this will select the scan closest to the double-click location (zoom in first for best results). Note that the scan list will be automatically scrolled to show the selected scan.

- By entering a scan number in the Scan\# text box, or typing the up or down arrow key with input focus in the Scan\# text box.

In the first two cases, select the "Spectrum" tab to see the relevant spectrum; in the third case, the spectrum will automatically be shown if you are viewing the chromatogram (Figure 3.1.3).

The scan list contains all the scans including MS1 and MS2 (MS/MS). Each MS/MS scan has the precursor $m / z$. Retrieval of charge state and activation of the precursor ions will be dependent on the raw data file format (Figure 3.1.3). Raw data from Thermo will likely contain information regarding the charge state and activation of the precursor ions. However, Bruker raw data does not provide this information. The user can manually specify the MS level of a scan by double-clicking on that scan. Doing this will show a dialog in which the MS level can be entered.

When the mouse cursor hovers over either a mass spectrum or chromatogram, the mouse location will be shown in the relevant data units $(\mathrm{m} / \mathrm{z}$ and intensity for a mass spectrum and retention time (RT) and intensity for a chromatogram) (Figure 3.1.3, Purple box). 

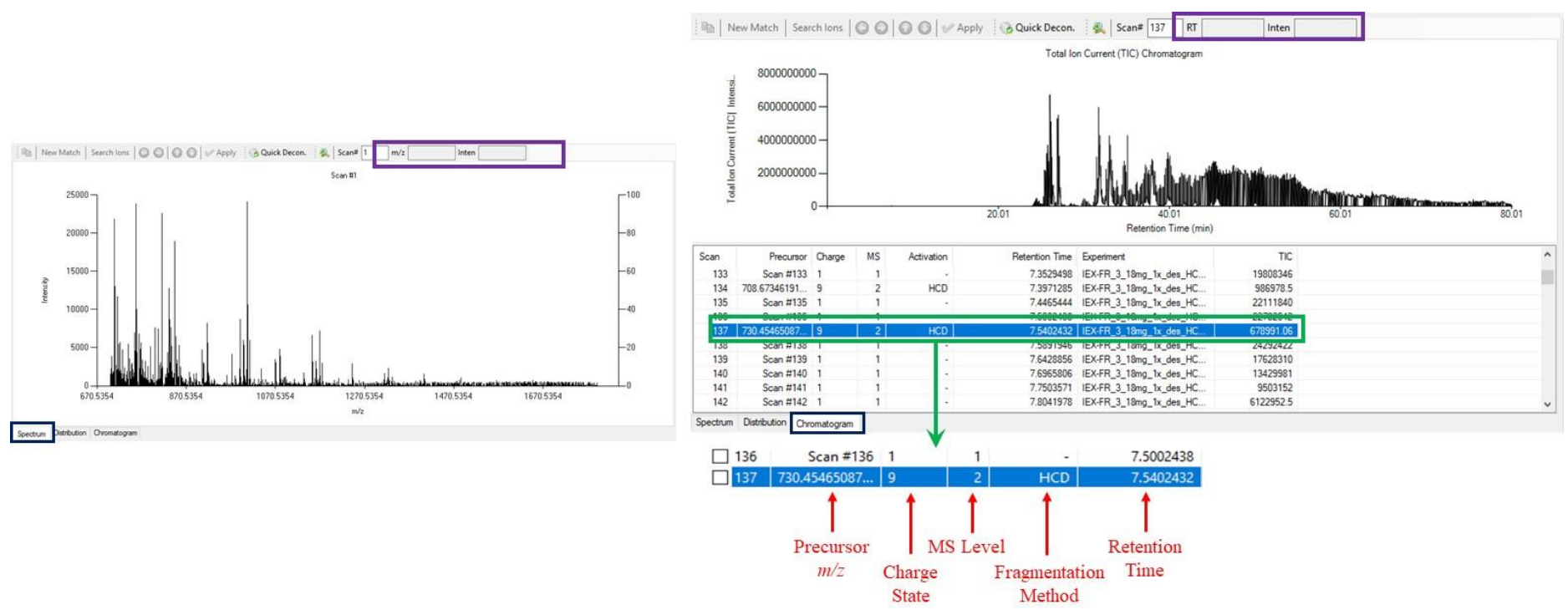

Figure 3.1.3 View windows for chromatograms and individual scans in the Results View panel.

\subsection{Process Data}

Data processing in top-down proteomics mainly involves centroiding, deconvolution, and database search. In the Discovery Mode, these data processing tasks are bundled together in the Process Wizard.

1. Click on "Process Data" and the database search wizard will open (Figure 3.2.1, left).

2. Select a deconvolution method, a database search method, and targeted protein sequence database file in .FASTA format (Figure 3.2.1, left).

3. Users can click on "Advanced" to change general settings and algorithm parameters for each algorithm (Figure 3.2.1, right). Specific parameter description for each algorithm can be found in the associated algorithm's website. Most algorithms have options for users to change the parameters such as the maximum and minimum values for fragment ion charge and mass. Additionally, users have the options to input fixed and variable post-translational modifications (PTMs). For search optimization, some algorithms such as TopPIC allow users to input the number of threads the algorithm can use. These parameters are accessible under "Advanced" $\rightarrow$ "Search" tab.

4. Click on the Start button to begin deconvolution and database search. MASH Explorer will attempt to detect the activation type from the data file. If the activation type is not detected, CID will be executed by default. User can specify the activation type to avoid errors.

* Please refer to the Software Installation Guide included in MASH Explorer installation (C:IProgram Files\Human Proteomics Program\MASH Explorerldoc) regarding the installation of each deconvolution and search software. 

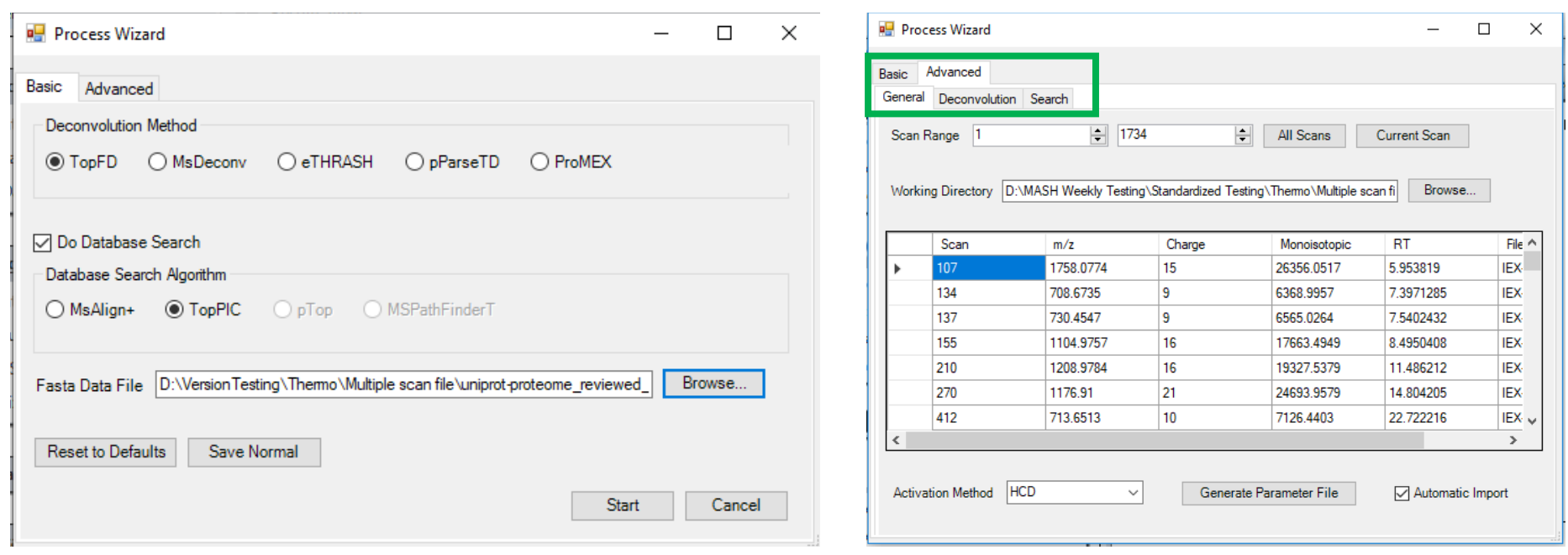

Figure 3.2.1 Basic and Advanced settings for Process Wizard in Discovery Mode

The search process will generate a folder under MASH_Workflows containing all the processing files (Figure 3.2.2). Do not open or delete any files in this folder before the search has completed. The processing folder will include the name of the data file, deconvolution algorithm, database search algorithm, and the time the workflow started (the format string of the folder is Filename_DeconvAlg_SearchAlg_YYYYMMDDHHMMSS). If the parameters are changed, the users can click "Save Normal" to allow these parameters to become default parameters for similar workflow. Reset to "Defaults" will allow the users to revert all the changed parameters to factory defaults.

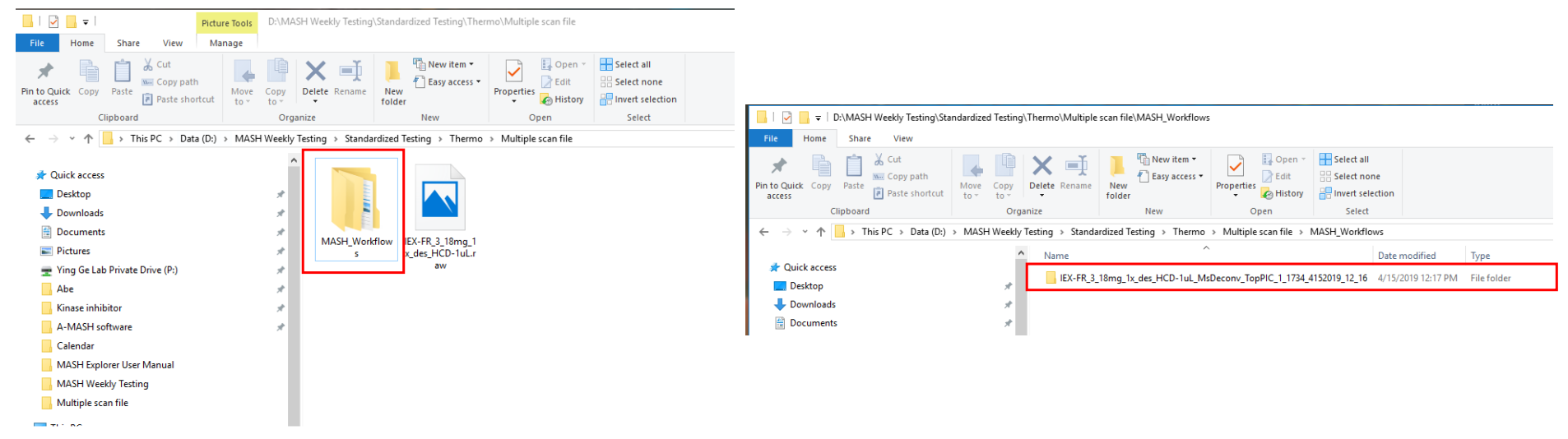

Figure 3.2.2 Folder created for data processing 
When the deconvolution/search workflow starts, the Workflow Manager will be opened (Figure 3.2.3).

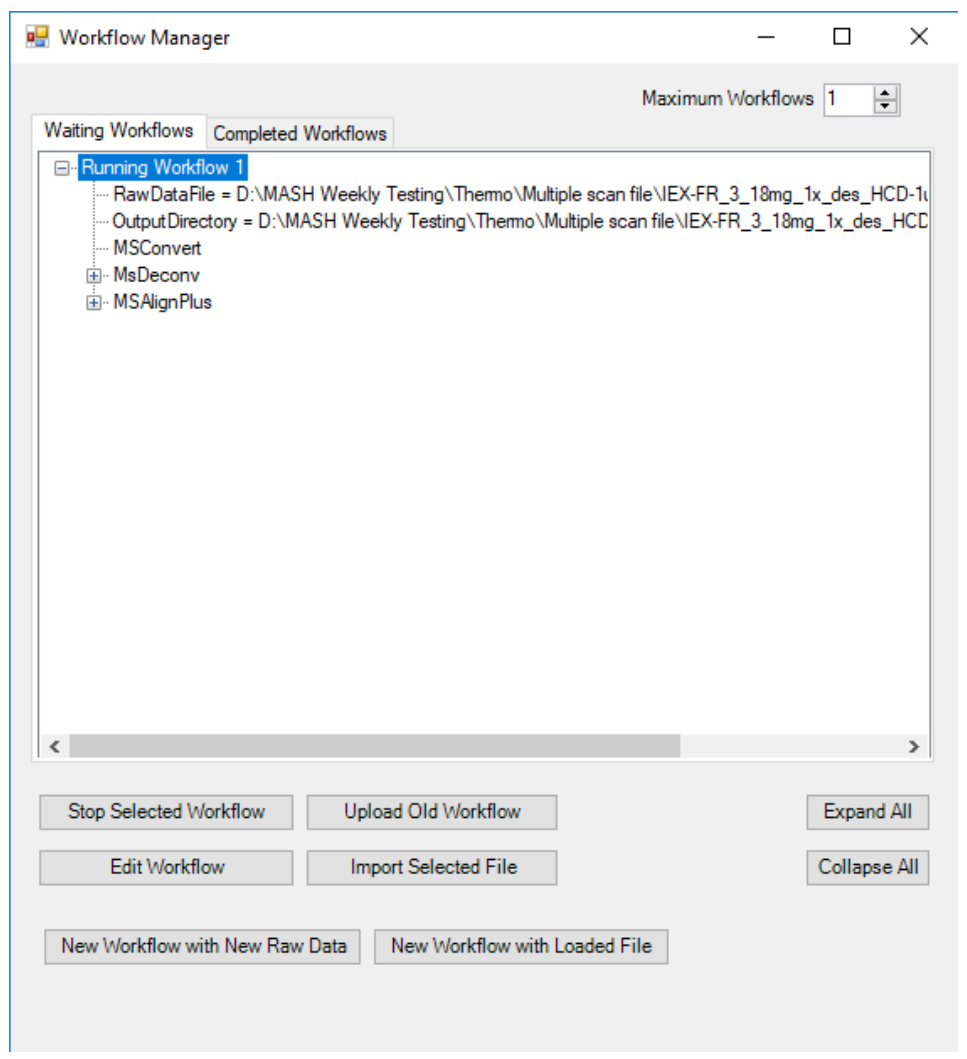

Figure 3.2.3 Interface for Workflow Manager

In the Workflow Manager, users can select several functions for a pending workflow:

- "Stop Selected Workflow" will delete the pending task in the queue.

- "Edit Workflow" allows users to modify parameters for pending and completed workflows (editing a workflow leaves the original workflow unchanged and creates a new workflow with the modified parameters).

○ Click on the "Edit Workflow" button to enable editing of a workflow. Users can edit both pending and completed (but not running) workflows.

- Triple click on a parameter to change the value (you can change multiple parameters sequentially).

○ Click "Done Editing/Re-run" to queue a new workflow with updated parameters.

○ Users can delete undesired pending workflows by selecting the workflow and clicking "Stop Selected Workflow".

- "Upload Old Workflow" will show a dialog to select the parameter file which can be found in a previous workflow folder (folders such as those shown in Figure 3.2.2).

- "Import Selected File" will import highlighted deconvolution or database search results file.

- The workflow progress can be monitored in both the Status Bar and LogBook. Users can click on "Completed Workflow" to view a finished workflow. If the Workflow Manager is closed, it can be reopened through Tools $\rightarrow$ Workflow Manager.

- Previously run deconvolution and database search results can be imported for analysis. Using the "Upload Old Workflow" button, users can import the Parameter.txt file. The analysis workflow will show under the "Completed Workflows" tab. After expanding the deconvolution and database 
search node, the deconvolution and database search result files can be located. Users can import these files by highlighting the file and clicking "Import Selected File" (Figure 3.2.4).
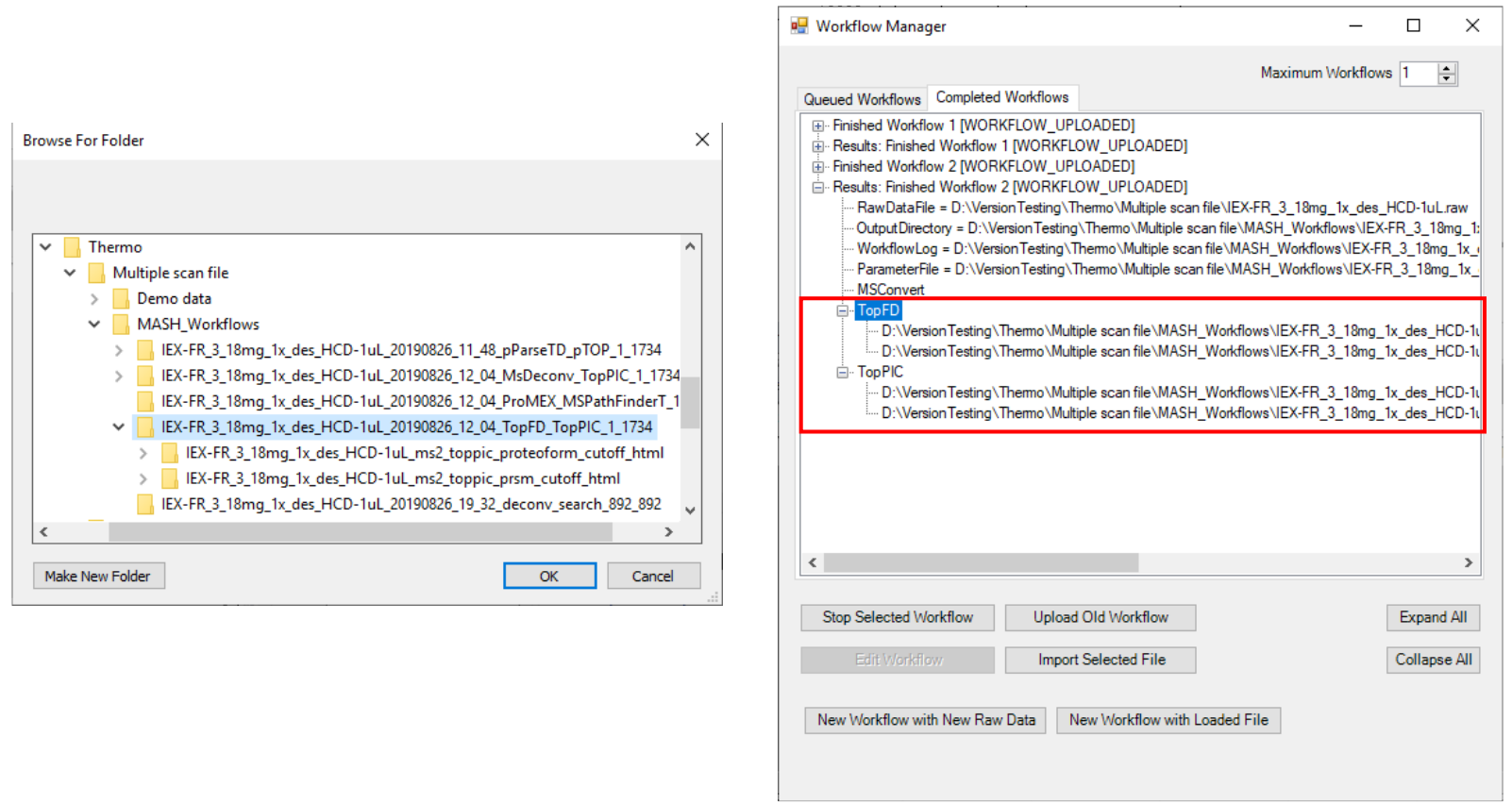

Figure 3.2.4 Uploading Processed Workflows

- Within the Workflow Manager, users can add new raw data for data processing and perform additional algorithm processing using the button "New Workflow with New Raw Data" and "New Workflow with Loaded File", respectively.

\subsection{Validate Data}

Workflow results can be imported by using Validate Data $\rightarrow$ Import Mass List to import deconvoluted mass list and Validate Data $\rightarrow$ Import Database Search to import protein search results (Figure 3.3.1).

Users can import the protein search results by clicking on entries in the "Protein Search Results", and the corresponding protein sequence will display in the Sequence Table panel (Figure 3.3.2). A detailed description of functions in the Sequence Table such as adding modifications and displaying fragment ions can be found in Section 6.2 Sequence Table of the user manual.

Users can also import the mass list containing fragment ion information which will display in the Mass List panel (Figure 3.3.2). A detailed description of functions in the Mass List, such as correction of fragment ion charge state and monoisotopic mass, is provided in Section 6.1 Mass List and Results View of the user manual. 

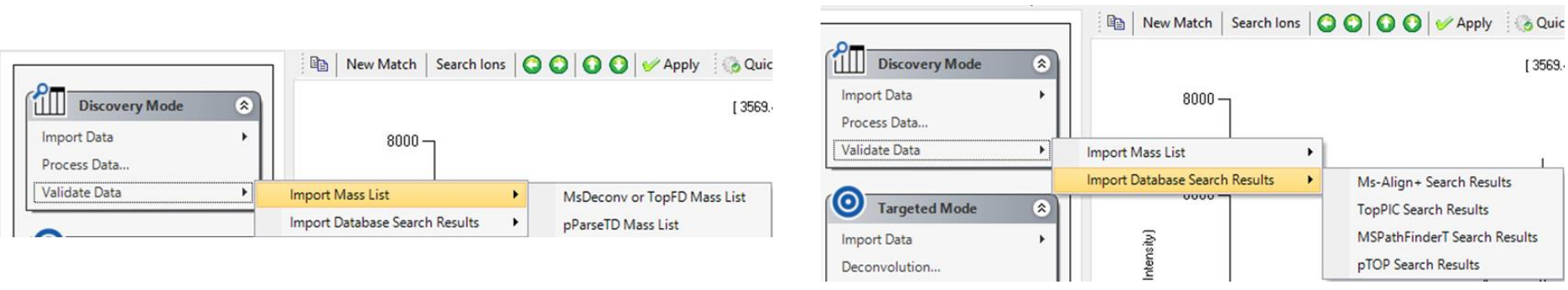

Figure 3.3.1 Menus for Validate Data
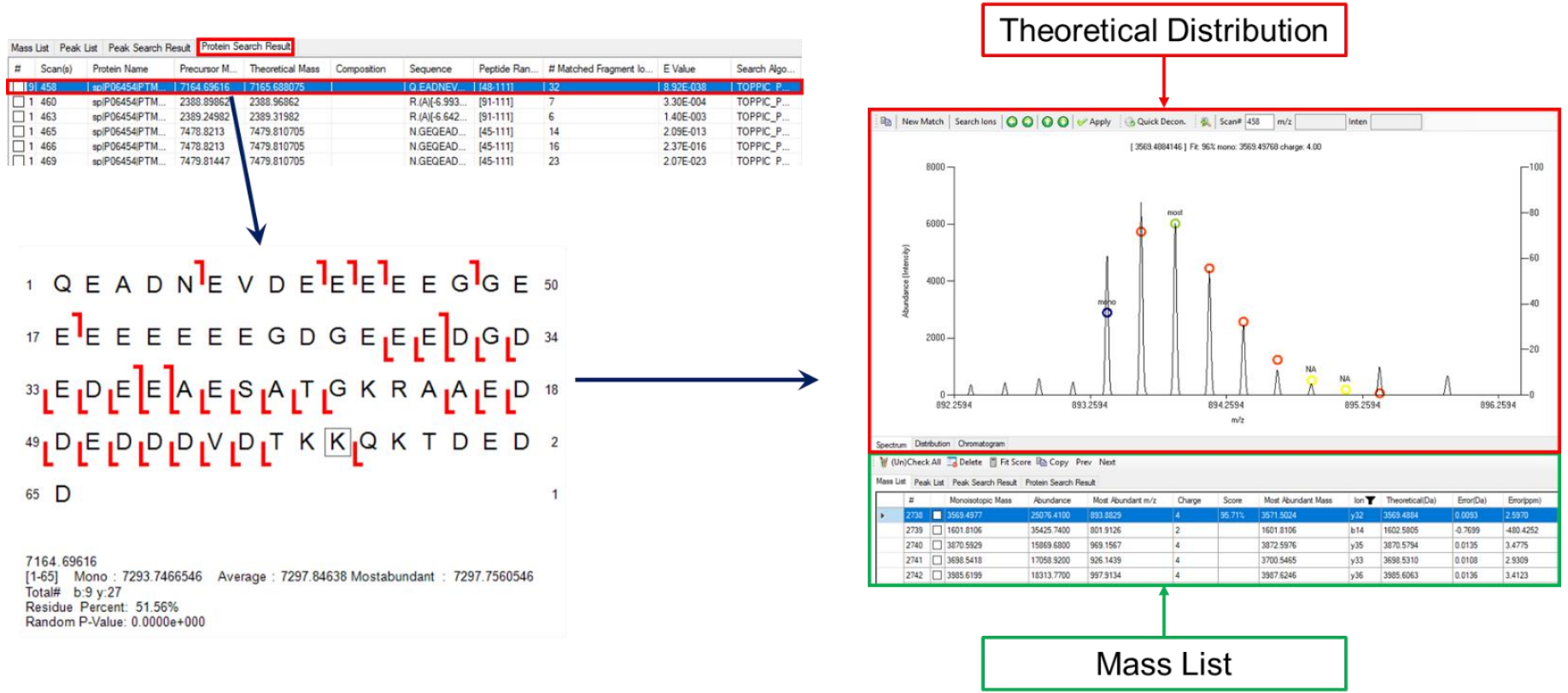

Figure 3.3.2 Analysis of protein sequence and fragment ion

\section{$\underline{\text { Automatic Import }}$}

For users' convenience, the mass list and protein sequence after database search are automatically imported. However, when users run more than one analysis, the automatic import function will stack one after another. Therefore, it is recommended to turn off the automatic import function when running more than one LC-MS/MS analysis. To disable automatic import, uncheck the automatic import checkbox in the "Advanced/General" tab (shown in the right half of Figure 3.2.1, checkbox at the bottom right) 


\section{Targeted Mode: Protein Identification and Characterization from MS/MS Data}

Contrary to Discovery Mode, our team developed a Targeted Mode workflow for analysis including comprehensive protein characterization. This workflow includes data import, spectral deconvolution to identification of fragment ions, database search based on identified ions, and finally protein characterization. This workflow aims to perform identification of fragmentation ions that describe the protein sequence and identification/localization of PTMs of a target protein sequence.

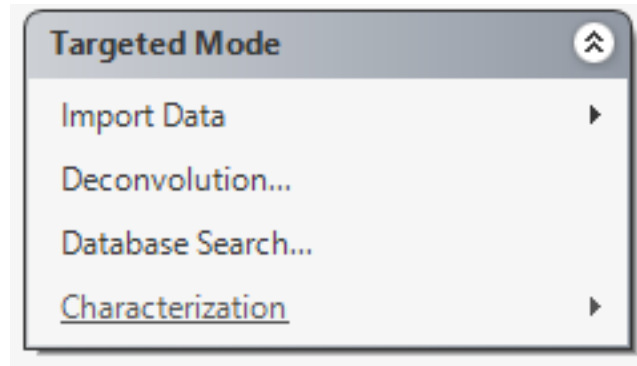

Figure 4.0.1 Targeted Mode button layout

\subsection{Import Data}

Similar to the "Import Data" in Discovery Mode, "Import Data" in Targeted Mode will direct users to import data into the software. Users can follow these steps for data import:

1. Click on "Import Data" in the Targeted Mode tab under the Workflow and Parameters panel to add a data set (Figure 4.1.1).*

2. Click on the option that represents the raw data file format to be uploaded (Figure 4.1.1). The current version of MASH Explorer supports Thermo (.raw), Bruker (.baf), Bruker data output in ASCII format (.ascii), and universal file formats (mzXML and MGF).

\begin{tabular}{|l|l|l|}
\hline Targeted Mode & (a) & \\
\hline Import Data & Thermo Finnigan Raw Data... \\
\hline Deconvolution... & MzXML Data Files... \\
Database Search... & Bruker Ascii File Import... \\
Characterization... & Bruker Baf Data File... \\
\hline & & MGF Data File... \\
\hline
\end{tabular}

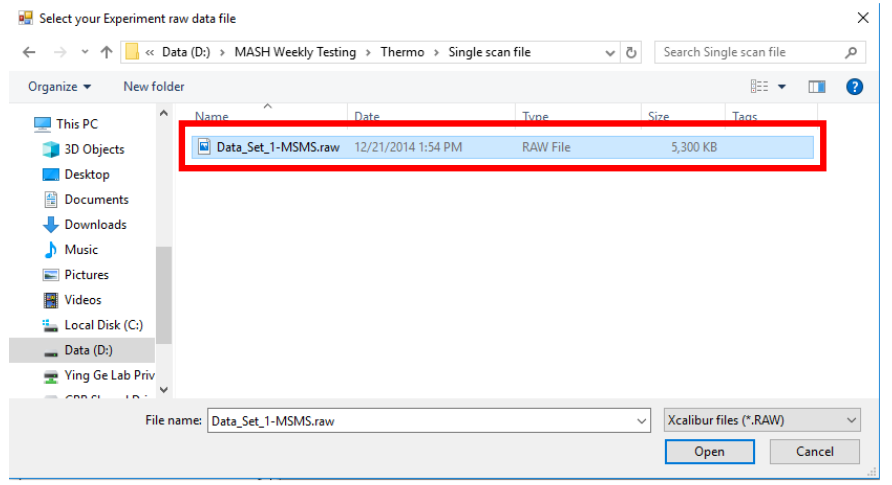

Figure 4.1.1 Data Import

* The user can also choose to drag and drop the data file to import into the software. 
Experimental data acquired from different instruments should be handled differently. Broadly speaking, there are two types of instruments: those that perform spectral averaging/summing during acquisition and those that perform averaging/summing post-acquisition.

1) Instruments that perform spectral averaging during acquisition include FT-ICR-type instruments including Thermo Finnigan Ultra and Bruker SolariX. The raw data normally has one single scan and can be processed directly by MASH Explorer.

2) Instruments that perform post-acquisition spectral averaging/summing include LC-MS/MS. For example, users inject a solution containing a single protein and use the LC to deliver the solution to the mass spectrometer. The resulting raw data have multiple scans and each scan contains fragment ion of the same protein. This type of data requires further processing prior to data import to MASH Explorer.

a. Thermo instruments: Xcalibur software can perform spectral averaging natively and the resulting spectrum can be exported in the RAW format.

b. Bruker instruments: DataAnalysis can perform spectral averaging and the resulting spectrum can be output as ASCII format.

c. Other instruments and general method: ProteoWizard has a filter function "Scan Summing" for spectral averaging, and the summed spectrum can be exported in mzXML format. Ideally, the output mzXML format has only one scan (Figure 4.1.2).

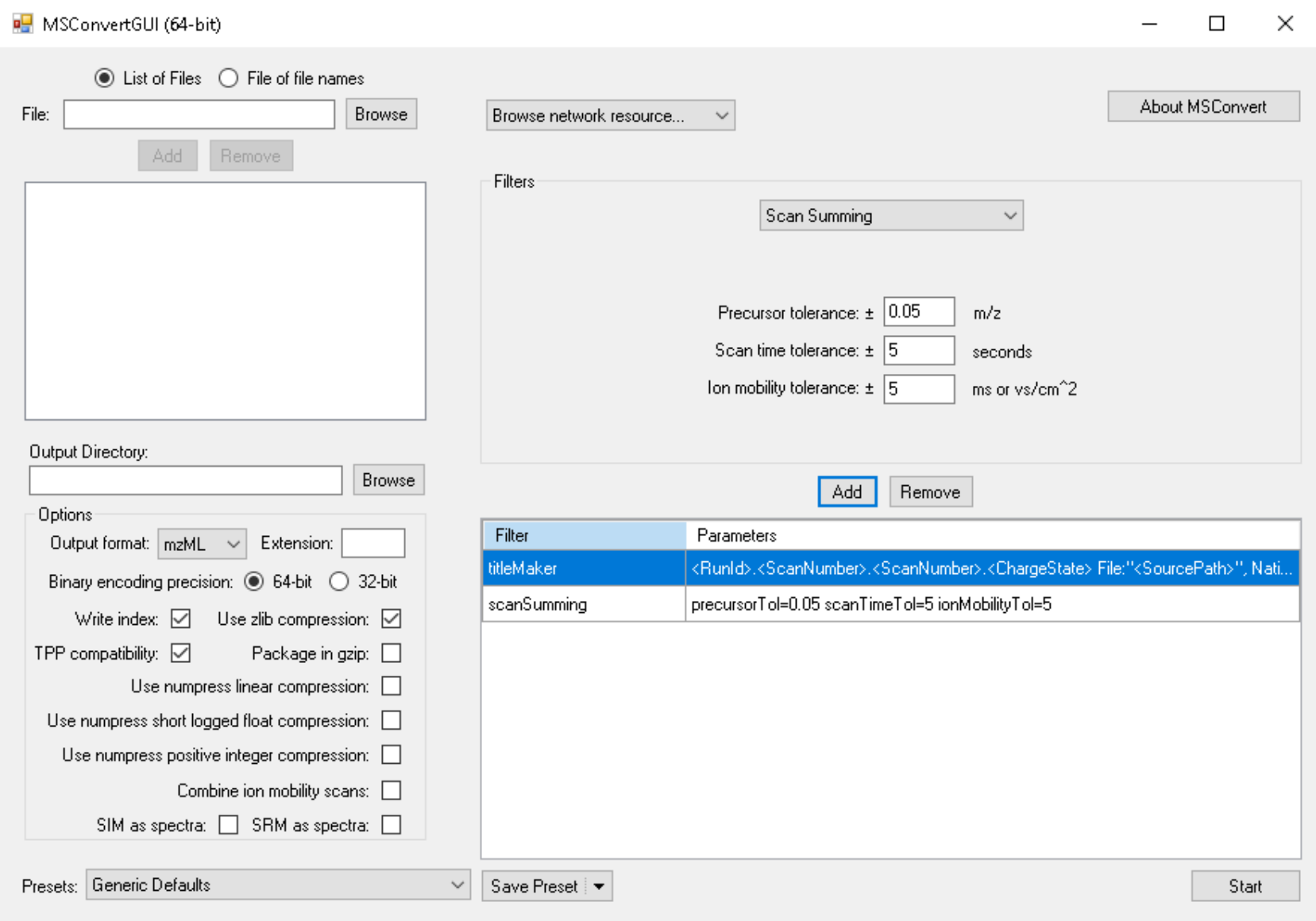

Figure 4.1.2 Scan Summing 


\subsection{Deconvolution}

Spectral deconvolution is the process of identifying fragment ion peaks and calculating their charge states, monoisotopic mass, most abundant mass, intensities, and other parameters. Our software currently offers three tools for spectral deconvolution in Targeted Mode: enhanced-THRASH (eTHRASH), TopFD, and MS-Deconv. Each of these algorithms can be automatically run by MASH Explorer using the Process Wizard (Figure 4.2.1).

Starting the deconvolution processes will initiate the Workflow Manager. When the calculation is finished, the calculated mass list from each algorithm will be automatically imported and displayed in the Mass List panel (Figure 4.2.1). Users can click on each fragment ion entry for viewing. The detailed functions of the Mass List are described in section 6.1 Mass List and Results View of the user manual.

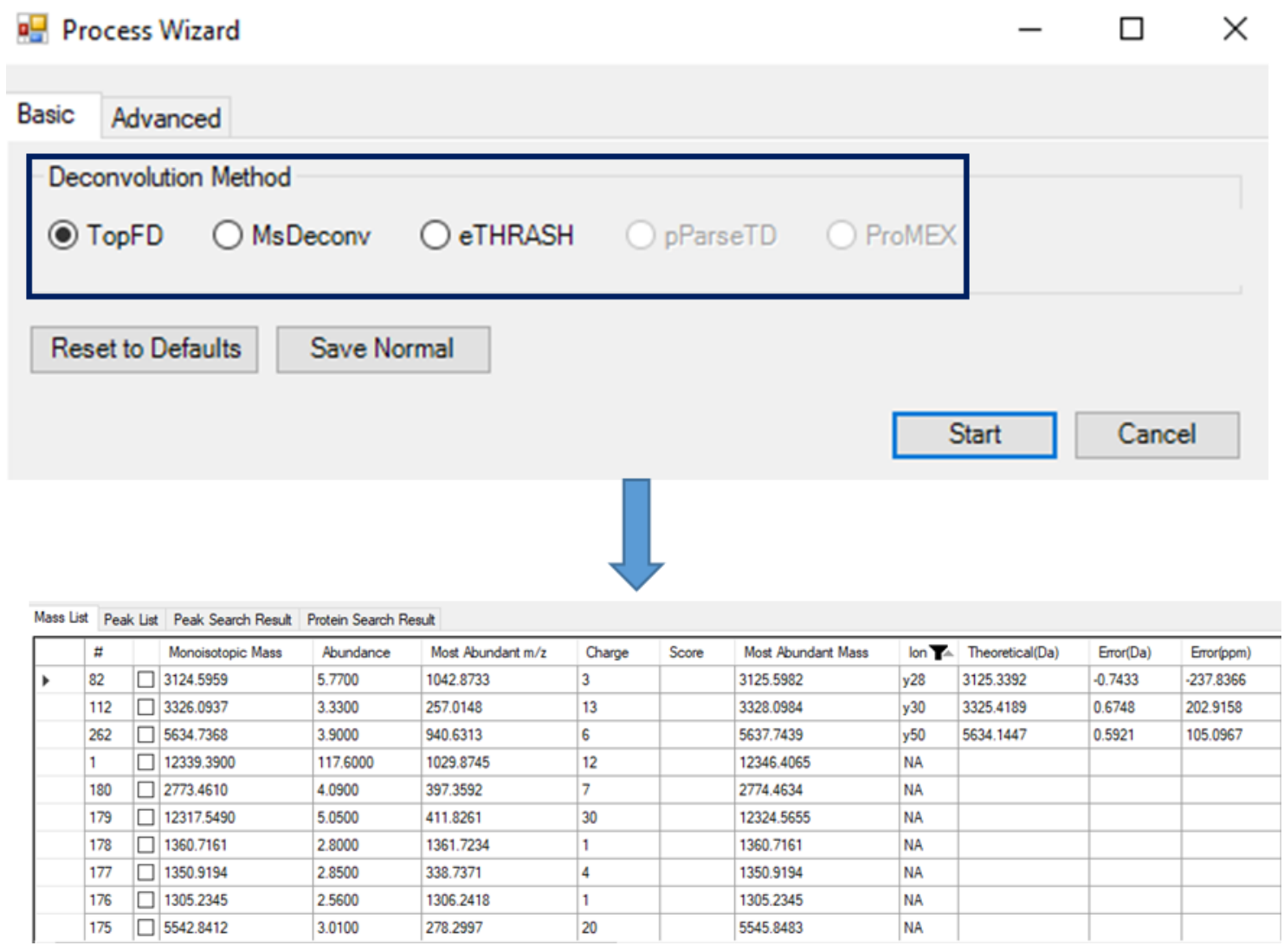

Figure 4.2.1 Basic interface of Deconvolution Process Wizard and Populated Mass List

\section{Notes on spectral deconvolution}

1. The current version of MASH Explorer supports TopFD, MS-Deconv and eTHRASH for spectral deconvolution in Targeted Mode.

2. eTHRASH, TopFD and MS-Deconv support MS deconvolution. MS-Deconv requires users to input the correct charge state of the precursor ion. User can input the ion charge state shown in Figure 4.2.2. 


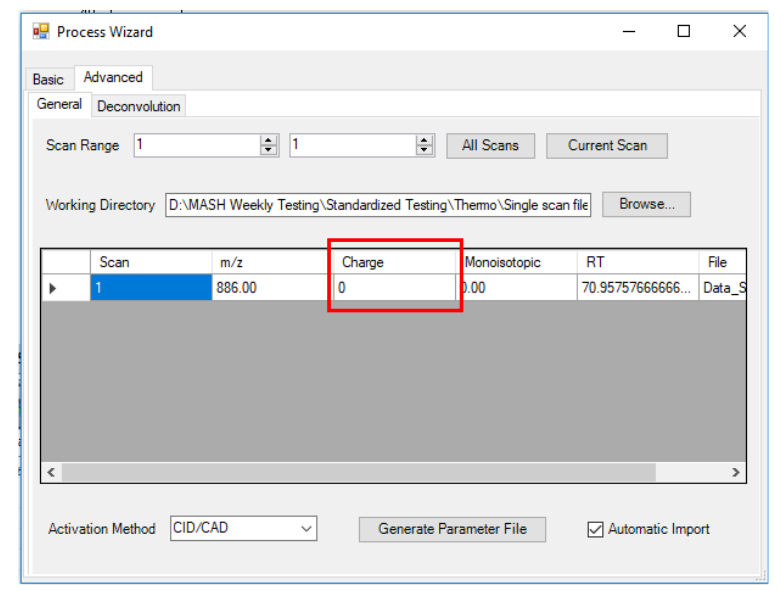

Figure 4.2.2. Process Wizard for Deconvolution.

3. The Quick Decon. button in the Results View can be used as a quick tool for spectral deconvolution. It supports both TopFD and eTHRASH, and the parameters can be configured under Tools $\rightarrow$ Quick Decon. Parameters. Details can be found in Section 7.4 Tools.

4. The current version of MASH Explorer cannot process MS/MS scans without an MS2 designation. This function will be included in the next public release. Users can manually edit the MS level of a scan by double-clicking on that scan in the chromatogram view (See Figure 3.1.3).

\subsection{Database Search}

Our workflow has incorporated algorithms that use fragment ion (MS/MS) information for protein identification. When the fragment ion selection is completed, the fragment ions can be searched against the database for protein identification. Users can choose one of the supported database search algorithms including MS-Align+ and TopPIC, and upload the target database (Figure 4.3.1). Click "Start" to begin the database search process and similar to other processes, the Workflow Manager will be invoked to handle algorithm execution.

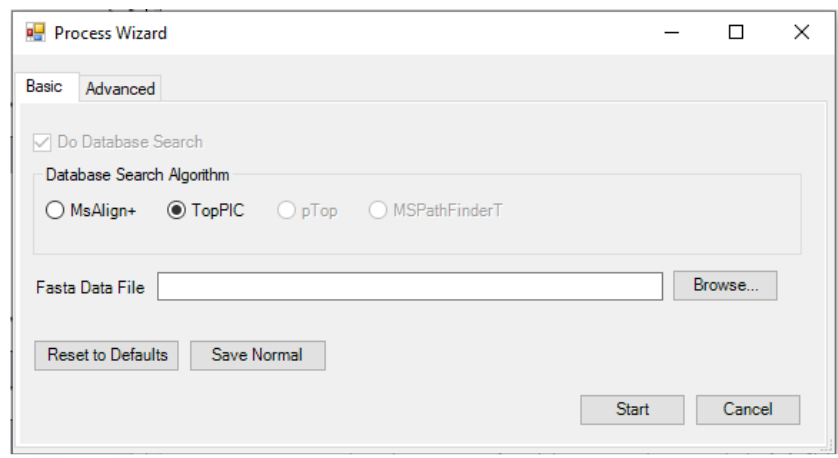

Figure 4.3.1 Basic Interface of Process Wizard in Search mode

For the Targeted Mode, it is recommended for the users to provide as much information as possible, as extraction of information from the MS/MS files is harder compared to the LC-MS/MS data. In the "Advanced" settings, 
users can enter the charge state of the precursor and the fragmentation activation method (Figure 4.3.2). This information will help substantially in file parsing processes prior to database search.

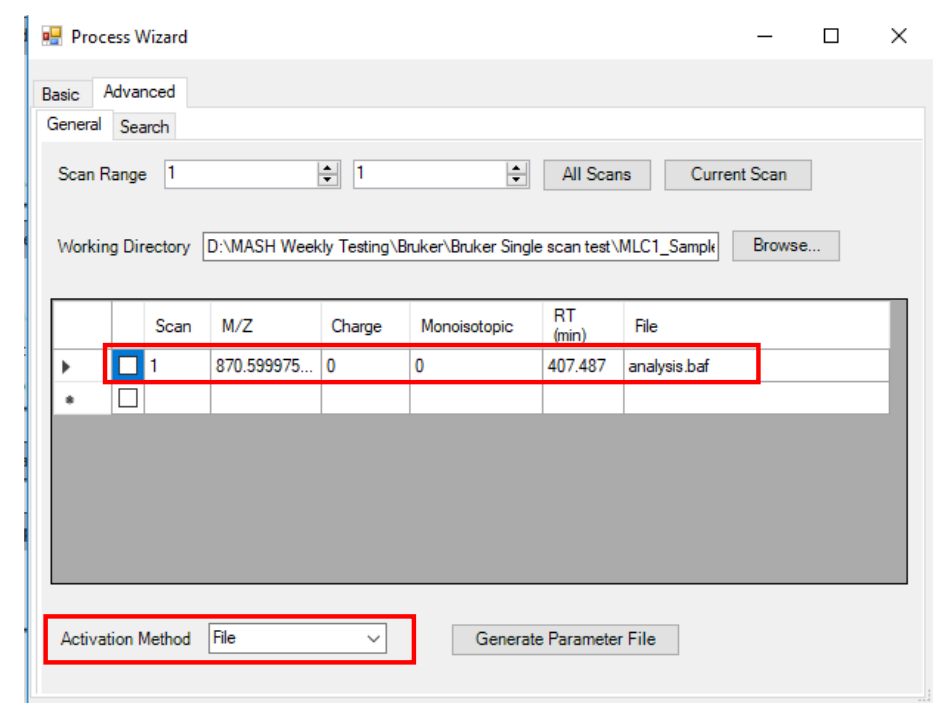

Figure 4.3.2 Advanced Settings for Database Search Wizard

\section{$\underline{\text { Notes on database search }}$}

1. The current version of MASH Explorer supports MS-Align+ and TopPIC for database search in Targeted Mode.

2. It is recommended that users input the charge state of the precursor ion and the activation method of the file in the specified locations in the Process Wizard shown in Figure 4.3.2. This information will help substantially in successful file parsing processes.

\subsection{Characterization}

The Characterization menu includes "Import Database Search Results" that imports the sequence from the database search and "Paste Your Sequence" which allows users to copy the sequence to the Sequence Table (Figure 4.4.1). A detailed description of the Sequence Table panel is in Section 6.2 Sequence Table. For database search list import, please refer to Section 3.3 Validate Data under Discovery Mode.

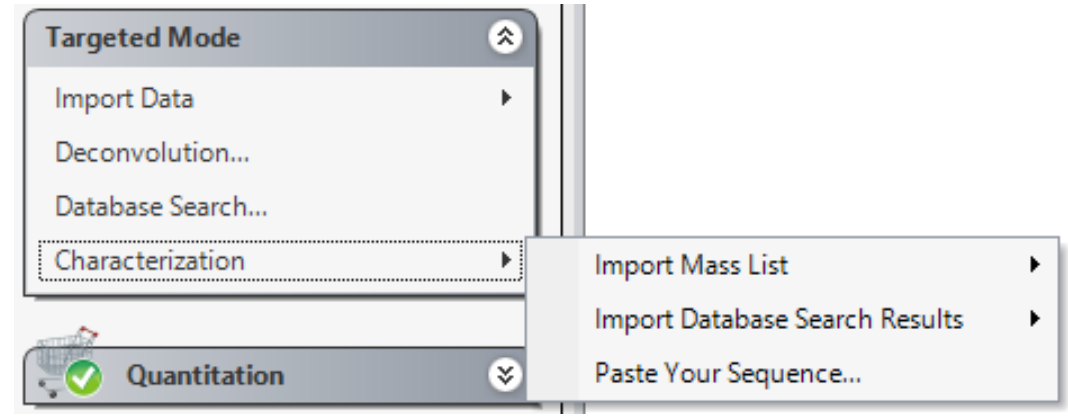

Figure 4.4.1 Menu for Characterization 
For manually pasting a protein sequence, click on "Paste Your Sequence" and the Paste Sequence Information window will be opened. Paste the sequence and click OK and the pasted sequence will be displayed in the Sequence Table (Figure 4.4.2).

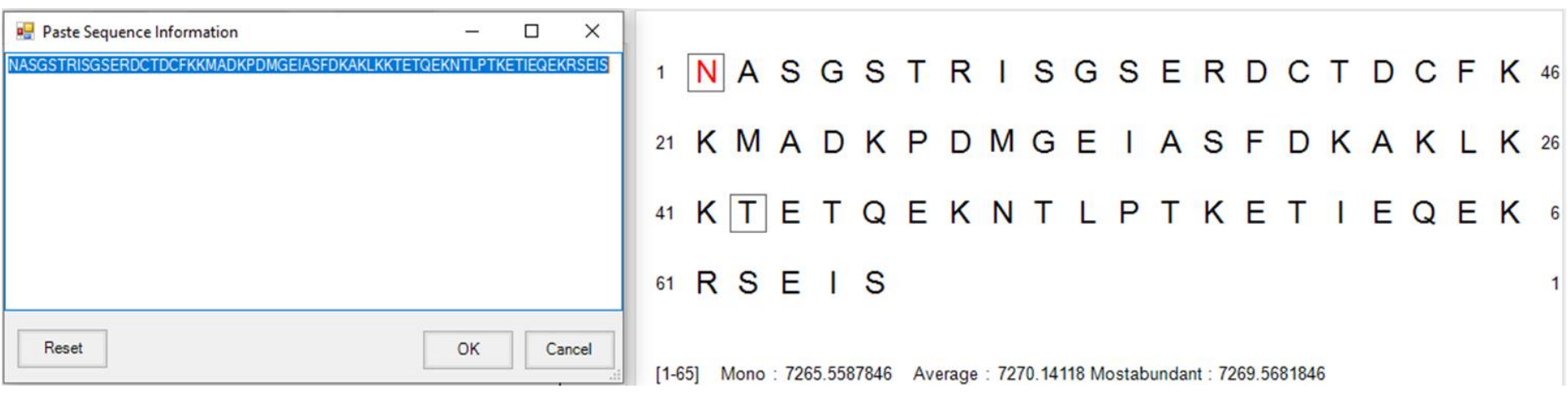

Figure 4.4.2 Pasting a protein sequence 


\section{Data Reporting}

This section of the manual introduces the functions in Data Reporting including Save Files and Save Images (Figure 5.0.1). These functions allow users to save the verified fragment ion list, enabling users to perform further processing of spectrum and sequence table images using other professional graphic software.

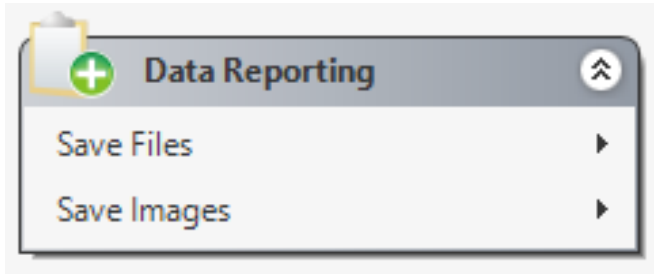

Figure 5.0.1 Data Reporting button layout

\subsection{Save Files}

Users can save their mass list and sequence table information in the .xml format which is associated with the original raw data (Figure 5.1.1). The .xml file is dependent on its original raw data, so it is recommended users place the saved .xml file in the same folder as the raw data.

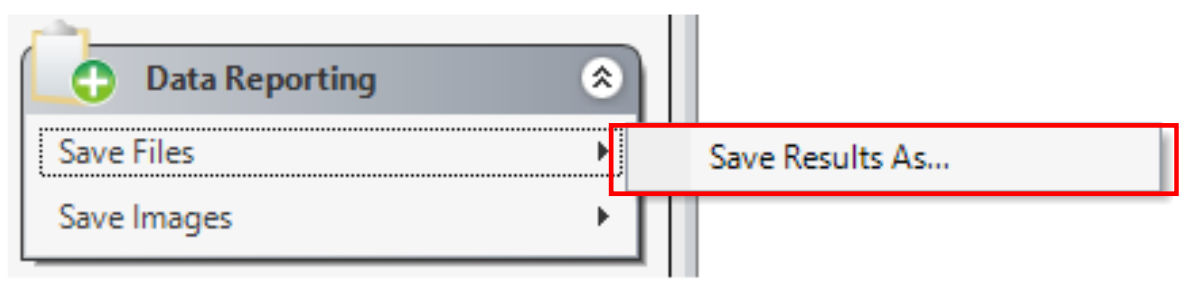

Figure 5.1.1 Save Files

To re-open the saved data, go to File $\rightarrow$ Open Saved Results. Since the .xml file needs its raw data for reference, users will be prompted to locate the raw data if the raw data is not found within the same folder.

\begin{tabular}{|llc|}
\hline File & Edit View Tools & Help \\
\hline & Open Sequence... & Ctrl+O \\
\hline & Open Saved Results... & Ctrl+Shift+O \\
\hline $\begin{array}{l}\text { Import Results } \\
\text { Close Experiment }\end{array}$ & $\mathrm{Ctrl+W}$ \\
\hline$\square$ & Save Results As... & $\mathrm{Ctrl}+\mathrm{S}$ \\
\hline & \\
\hline
\end{tabular}

Figure 5.1.2 Open Saved Results 


\subsection{Save Images}

A spectrum displayed in the Results View panel and sequence information shown in Sequence Table panel can be exported as a Microsoft Office object (Figure 5.2.1). This function enables users to further edit the images including changing the font, font size of the text and line width and color of the fragment symbols. The copied image can be transferred to Adobe Photoshop, Illustrator, or Microsoft PowerPoint for further processing. Elements of the graph can be ungrouped for further modifications.

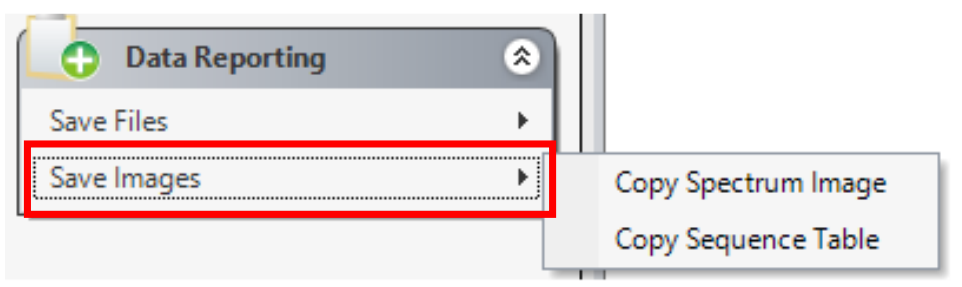

Figure 5.2.1 Menu for Save Images

In "Save Images", “Copy Spectrum Image” will copy the spectrum in the Results View panel (Figure 5.2.2).

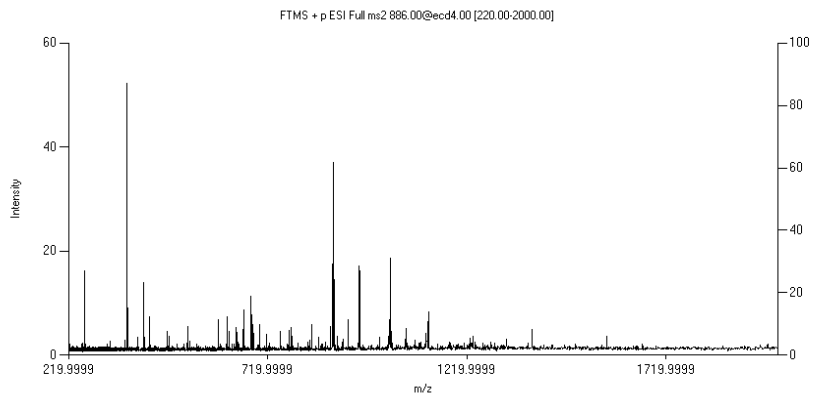

Figure 5.2.2 Example spectrum image as a Microsoft Office object

In "Save Images”, “Copy Sequence Table” will copy the spectrum in the Sequence Table panel (Figure 5.2.3).

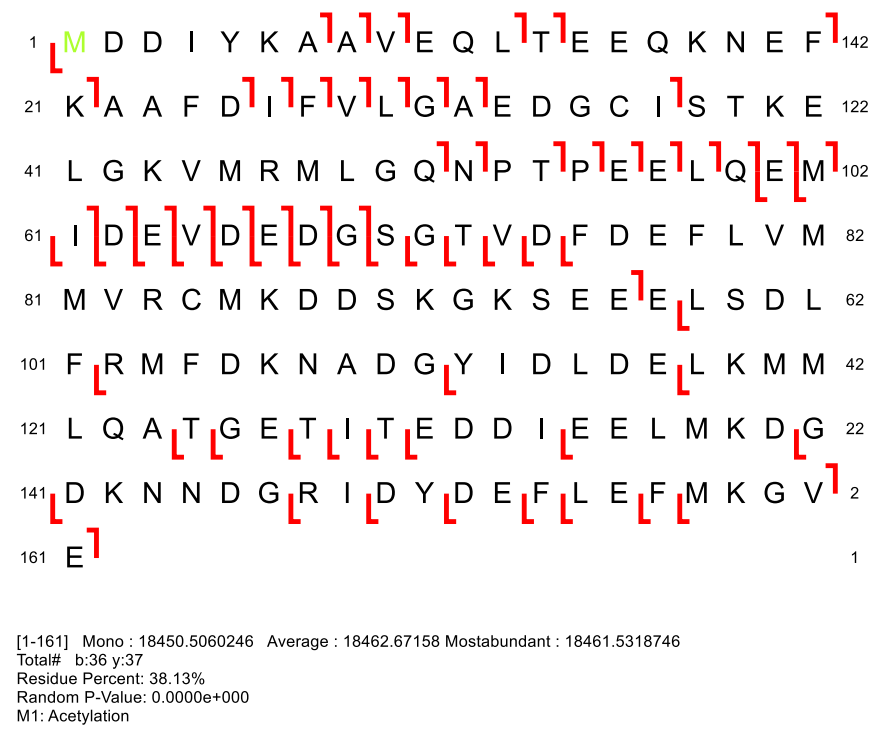

Figure 5.2.3 Example Sequence Table image as a Microsoft Office object 


\section{Visual Validation of the Computational Output}

This section of the manual will introduce the function in Mass List and Results View, Sequence Table and Ion Finder. These tools are essential for the protein characterization using MASH Explorer software.

\subsection{Mass List and Results View}

The Mass List provides an intuitive display combining the theoretical ion distribution and the actual experimental spectra shown in Results View. After deconvolution, users can click on each entry in the mass list and the spectrum will be zoomed in to the location of the targeted ion. The red circles represent the theoretical ion distribution. The blue and green circles represents the monoisotopic $m / z$ and most abundant $m / z$, respectively. Previously selected ions will have a light yellow background in the Mass List. The up/down arrow keys will change the selected ion in the Mass List.

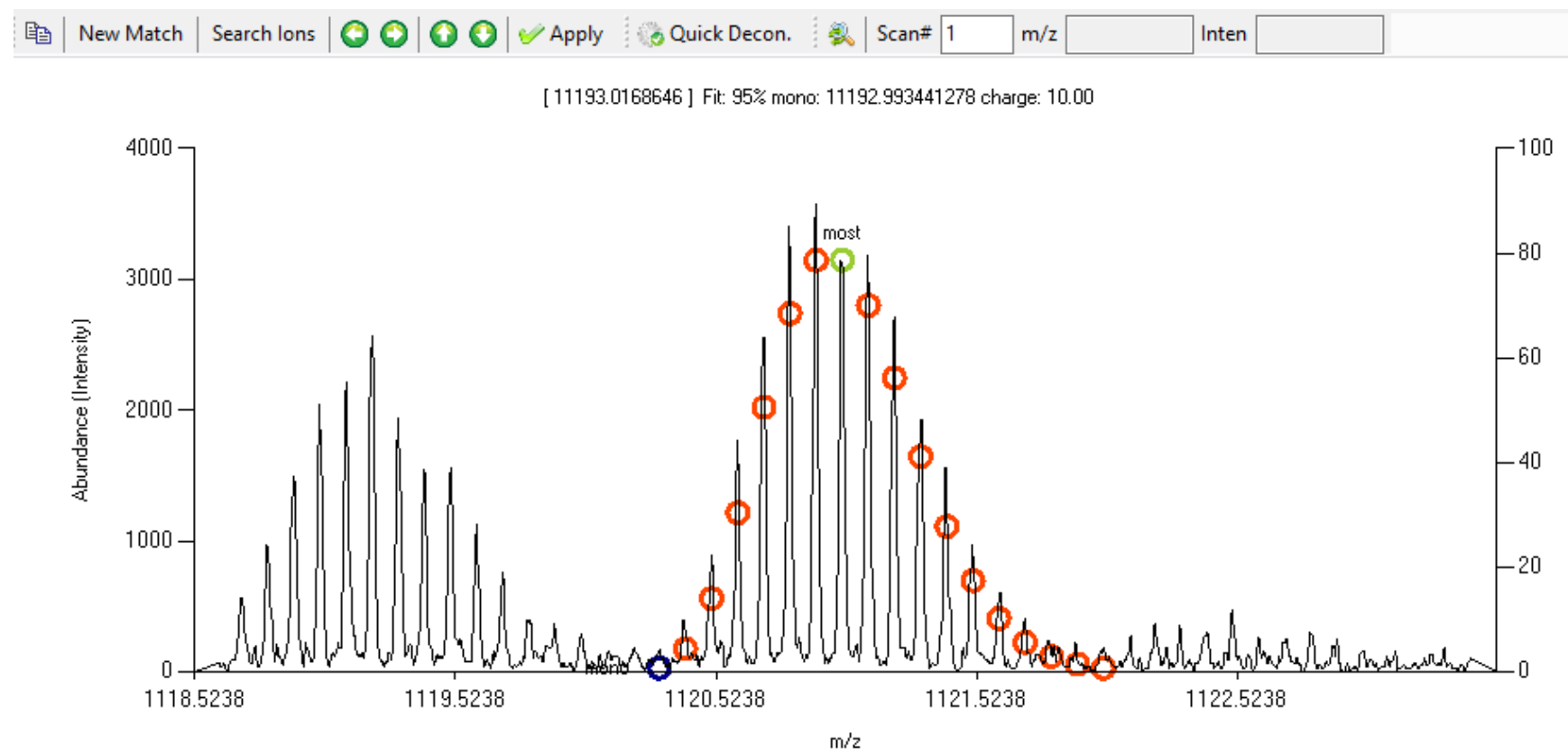

\begin{tabular}{|c|c|c|c|c|c|c|c|c|c|c|c|}
\hline \multicolumn{2}{|c|}{ Spectrum } & \multicolumn{10}{|c|}{ Distribution Chromatogram } \\
\hline \multicolumn{12}{|c|}{ W (Un)Check All $\rightrightarrows$ Delete Fit Score 踝 Copy Prev Next } \\
\hline \multirow[t]{7}{*}{ Mass List } & \multicolumn{2}{|c|}{ Peak List } & Peak Search Result & \multicolumn{8}{|c|}{ Protein Search Result } \\
\hline & $\#$ & & Monoisotopic Mass & Abundance & Most Abundant $\mathrm{m} / \mathrm{z}$ & Charge & Score & Most Abundant Mass & $\operatorname{lon} \boldsymbol{T}$ & Theoretical(Da) & Error(Da) \\
\hline & 1 & $\square$ & 18450.4480 & 4626.4756 & 1086.9759 & 17 & $97.00 \%$ & 18461.4766 & y161 & 18450.5060 & -0.0580 \\
\hline & 2 & $\square$ & 18452.4570 & 4626.4756 & 1087.0941 & 17 & $85.53 \%$ & 18463.4857 & NA & & \\
\hline & 3 & $\square$ & 7257.4756 & 4173.5986 & 1038.3619 & 7 & $97.32 \%$ & 7261.4862 & b64 & 7257.4892 & -0.0135 \\
\hline & 4 & $\square$ & 6914.3322 & 3528.9397 & 989.3414 & 7 & $97.93 \%$ & 6918.3426 & b61 & 6914.3512 & -0.0191 \\
\hline & 5 & $\square$ & 18432.4710 & 3995.7209 & 1085.9185 & 17 & $96.90 \%$ & 18443.4997 & b161 & 18432.4955 & -0.0244 \\
\hline \multirow[t]{3}{*}{$\triangleright$} & 6 & $\square$ & 11192.9934 & 3178.9695 & 1121.0079 & 10 & $95.24 \%$ & 11200.0118 & y97 & 11193.0169 & -0.0234 \\
\hline & 7 & $\square$ & 6670.2154 & 3046.3022 & 954.4676 & 7 & $98.80 \%$ & 6674.2258 & b59 & 6670.2267 & -0.0112 \\
\hline & 8 & $\square$ & 6670.2199 & 2810.5474 & 1113.3784 & 6 & $97.78 \%$ & 6674.2303 & b59 & 6670.2267 & -0.0068 \\
\hline
\end{tabular}

Figure 6.1.1 Result View and Mass List interactive display 
Users can change the following properties of the ion distribution to achieve better theoretical ion fitting to the experimental data:

1. Adjust the charge state of the theoretical distribution:

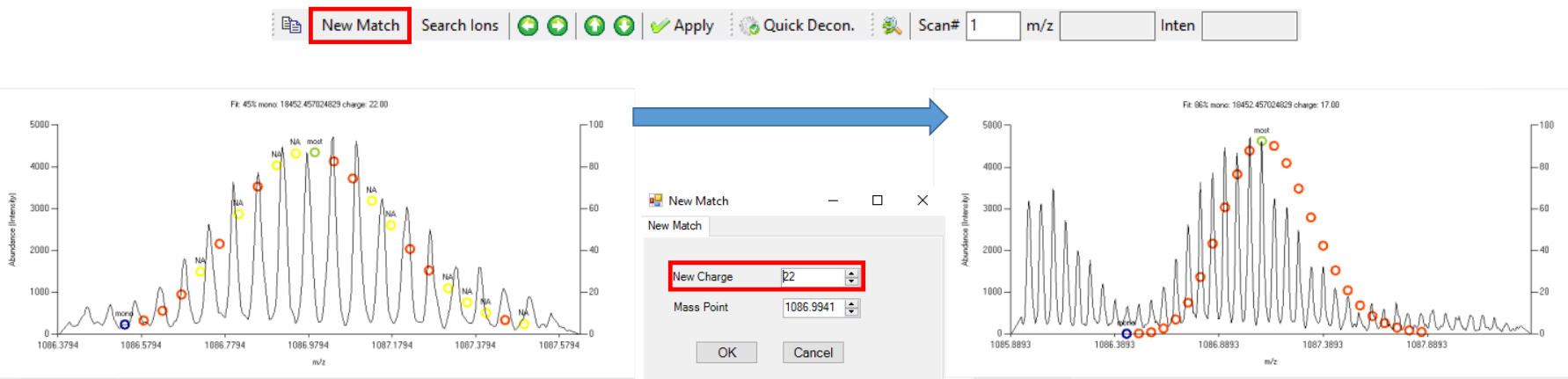

2. Add or subtract one isotopic unit of the theoretical ion distribution:

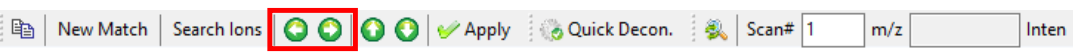
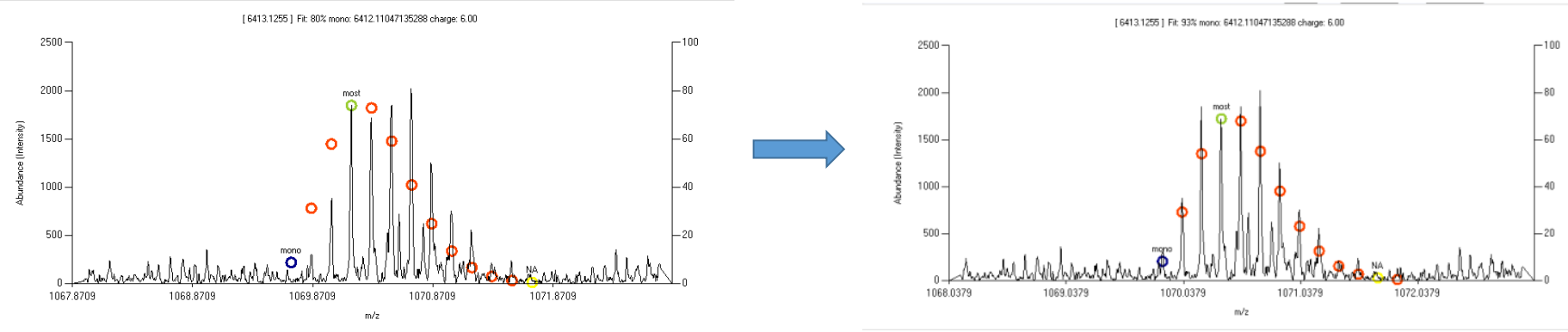

3. Increase or decrease the signal intensities:

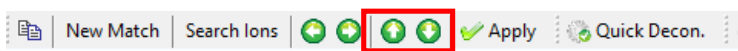

Scan\# $1, \mathrm{~m} / \mathrm{z}$ Inten
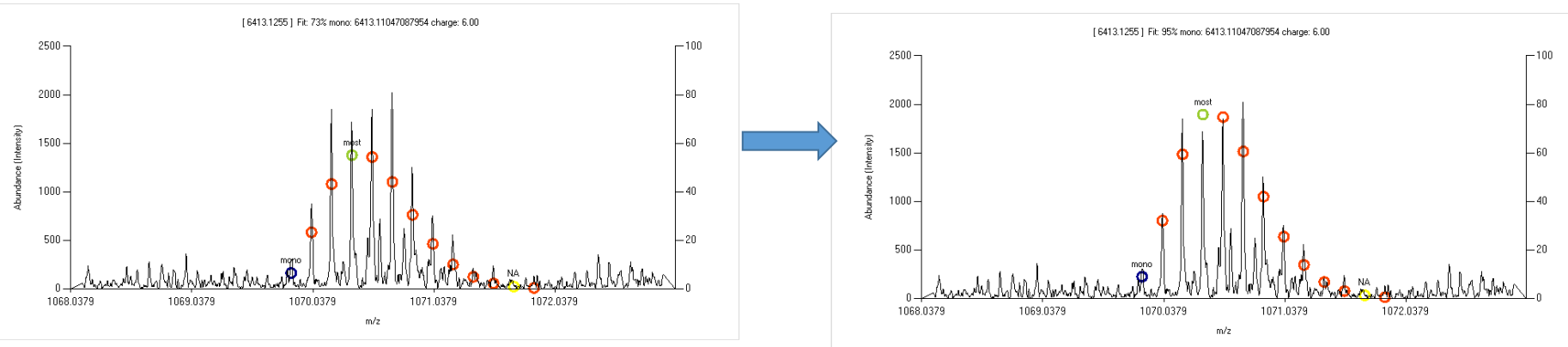

Clicking "Apply" will save the fragment ion adjustments.

The Mass List also allows users to sort the items by values in each column, such as monoisotopic mass, abundance, $\mathrm{m} / \mathrm{z}$, charge, score, most abundant $\mathrm{m} / \mathrm{z}$, ion designation, and so on (Figure 6.1.2).

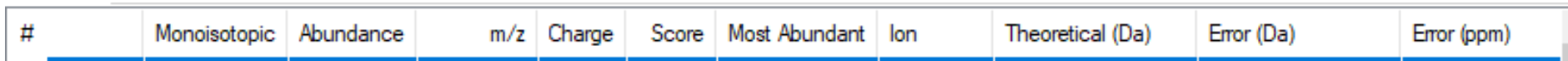

Figure 6.1.2 Mass List column items 
The tool bar in the Mass List panel includes buttons such as "(Un)Check All", "Delete", "Fit Score", "Copy", "Prev", and "Next" (Figure 6.1.3). These buttons interact with the checked item(s) in the Mass List.

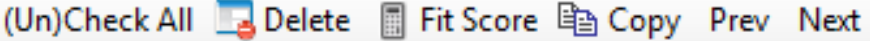

Figure 6.1.3 Mass List Tool Bar

- The "(Un)Check All” button will check or uncheck all the boxes in the Mass List.

- The "Delete" button will delete all items that are checked.

- The "Fit Score" button will calculate all the Score column in the mass list. The Score column describes the fitting between the experimental and theoretical isotopic distribution

- The "Copy" button will copy all items that are checked. The copied content can be pasted in processing software such as Notepad and Microsoft Excel.

- "Prev" and "Next" will move the previous or next scan for which Mass List entries exit.

\subsection{Sequence Table}

Once the Mass List is verified by the users, the Mass List can be matched with the theoretical fragment ions list generated by the user-imported sequence to show fragmentation mapping of proteins (Figure 6.2.1). The sequence can be imported via "Characterization" $\rightarrow$ "Paste Your Sequence" in Targeted Mode, or the "Modify Sequence" button in the Sequence Table panel (Figure 6.2.1).

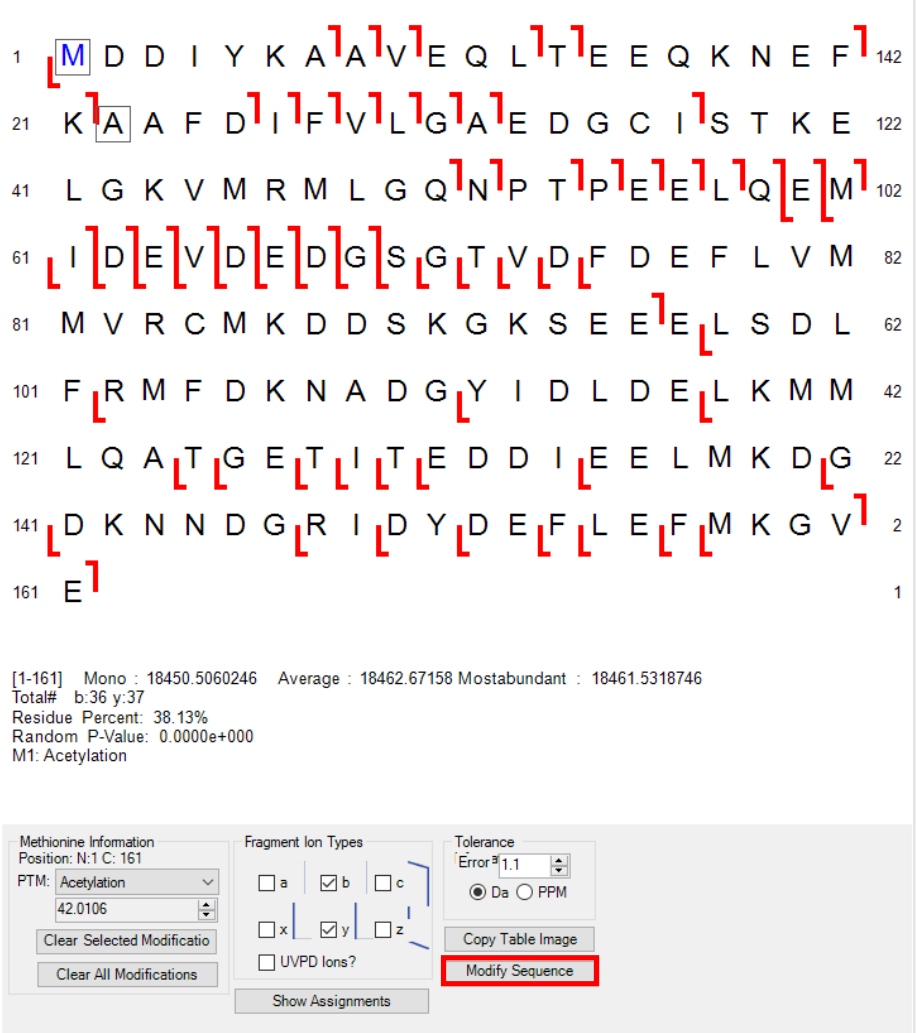

Figure 6.2.1 Sequence Table panel 
In the Modifications section, users can perform several tasks. To add a modification, the user needs to click on a specific amino acid. The table below shows the colors of different instances.

\begin{tabular}{|c|c|c|}
\hline & Not Selected & Selected \\
\hline w/o PTM & Black & Red \\
\hline w/ PTM & Green & Blue \\
\hline
\end{tabular}

There are preset modifications with mass changes such as phosphorylation $(+79.96 \mathrm{Da})$ and methylation $(+14.01$ Da). Users can also choose Custom to provide a custom molecular weight change specific for the analysis (See Figure 7.4.3). A modification can be removed by "Clear Selected Modification" and all the modifications can be removed by "Clear All Modifications".

In the Fragment Ion Types section, users can map the fragment ion in the Mass List to the sequence in the Sequence Table by selecting fragment ion types and clicking "Show Assignments". The lines between two amino acid codes suggested fragment ion detection. We currently support $\mathrm{a} / \mathrm{x}, \mathrm{b} / \mathrm{y}$ and $\mathrm{c} / \mathrm{z}$ fragment ion types (Figure 6.2.1). Additionally, the current version supports common UVPD ions such as $a+1, x+1$, and $y-1$.

In the "Tolerance Information" section, the fragment ion mapping can be manipulated by different tolerance values in both ppm and Da units.

Users can copy the image shown in Sequence Table by "Copy Table Image". The copied image can be pasted in either Photoshop or Microsoft PowerPoint for further processing.

\subsection{Ion Finder}

The Ion Finder tool allows users to perform targeted search for fragment ions against a user imported sequence. In the Theoretical Data from the Sequence Table panel, the monoisotopic mass of different ion types is calculated based on the sequence with modifications provided in the Sequence table. Double-clicking a selected entry will open up the Ion Finder tool for the specific fragment number (Figure 6.3.1).

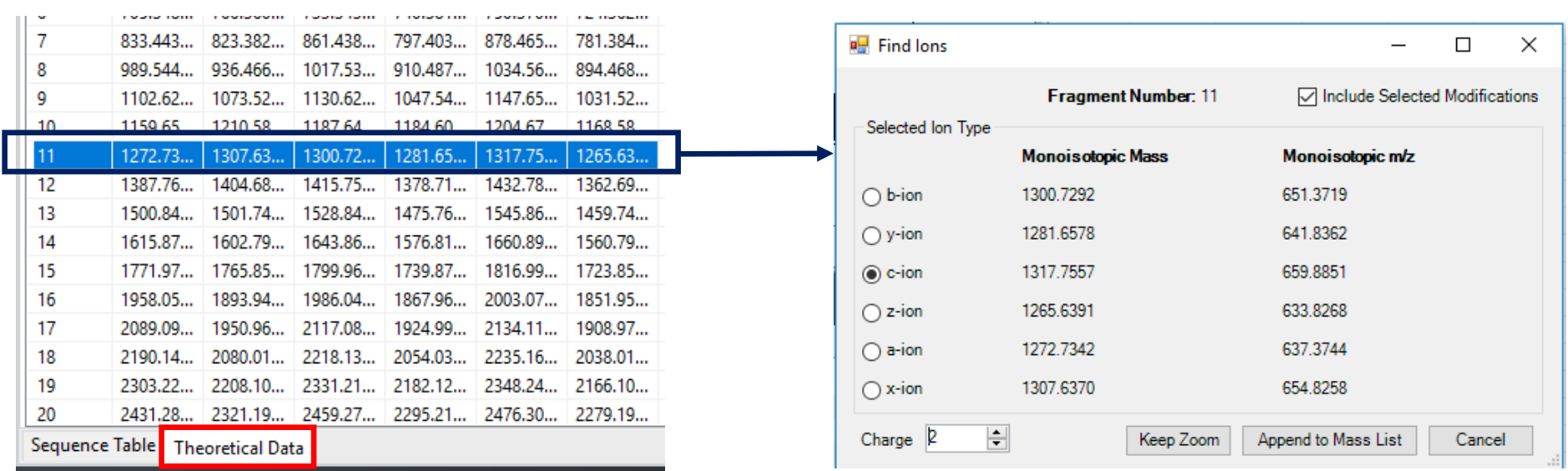

Figure 6.3.1 Ion Finder tool 
Users can select the fragment ion type for their experiment by clicking at selected radio buttons. Users will also be able to choose to include or exclude selected modification implemented in the sequence. The Ion Finder tool allows users to navigate different charge states of the fragment ion to examine possible experimental fragment ions by zooming at the targeted $\mathrm{m} / \mathrm{z}$ region calculated based on the monoisotopic mass and charge state of the fragment ion. The spectrum will change accordingly with changes to parameters such as selected ion type, modifications, and charge state (Figure 6.3.2), with theoretical value shown as red circles.

Users can stay zoomed-in at the current spectral location by clicking "Keep Zoom". If the users find a real ion, clicking "Append to Mass" List will add the ion information, including modification information, as a new entry at the bottom of the Mass List (Figure 6.3.2).

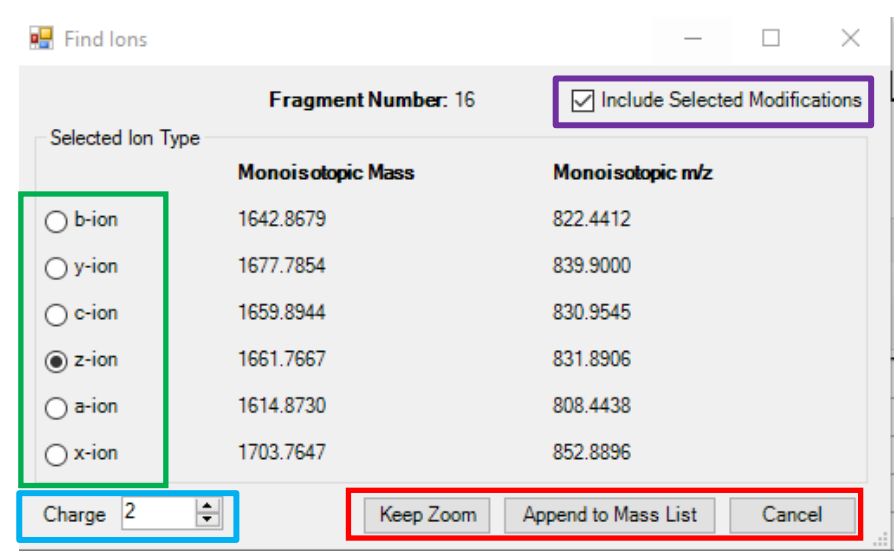

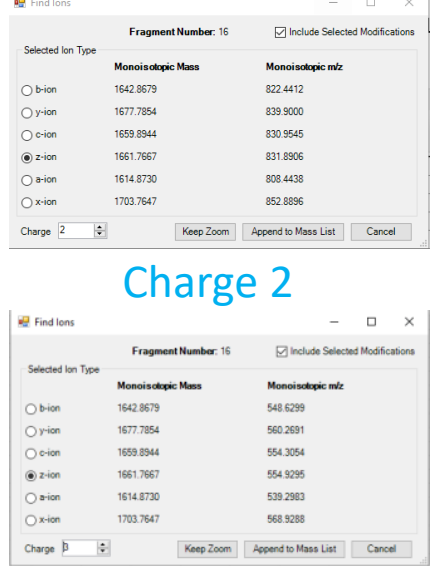

Charge 3
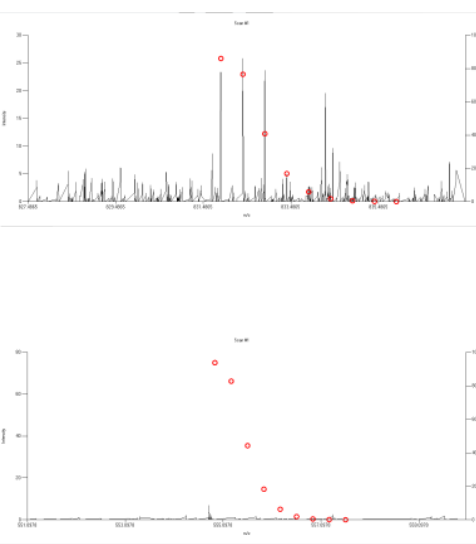

Figure 6.3.2 Functions for Ion Finder Tools 


\section{Tool Bar}

The tool bar in the top left corner of the window allows users to access some of the basic functions of the software.

File Edit View Tools Help

Figure 7.0.1 Tool Bar button layout

\subsection{File}

Items under "File" include "Open Sequence", “Open Saved Results", "Import Results", "Close Experiment", "Save Results As", and "Exit" (Figure 7.1.1).

\begin{tabular}{|c|c|c|c|c|}
\hline File & Edit & View & Help & \\
\hline \multirow[t]{4}{*}{$\nabla$} & Open & equence... & $\mathrm{Ctrl}+\mathrm{O}$ & \\
\hline & Open & aved Results... & Ctrl+Shift +0 & \\
\hline & Impor & Results & & • \\
\hline & Close & (periment & $\mathrm{Ctrl}+\mathrm{W}$ & \\
\hline \multirow[t]{2}{*}{ 目 } & Save $R$ & sults As... & $\mathrm{Ctrl}+\mathrm{S}$ & \\
\hline & Exit & & & \\
\hline
\end{tabular}

Figure 7.1.1 Menus for File option

- "Open Sequence" allows users to import a fasta sequence file. This function will be deactivated in future versions.

- The "Open Saved Results" button provides users a method to open saved .xml results. This function is related to "Save Results As" button described below.

- The "Import Results" tab has options for users to import both an ion mass list (in .msalign format) and a protein search result (in related database search algorithm format).

- "Close Experiment" will close the current imported data set.

- "Save Results As" will output an xml file that includes the information from the Mass List and Sequence Table. The xml file cannot be opened by itself as it is dependent on the corresponding raw data. If a raw data file is not found, the program will prompt the user to specify the raw data for reference.

- "Exit" will close the software application. 


\subsection{Edit}

Items under "Edit" include "Copy Checked Mass(es)", "Copy Sequence Table", "Copy Spectrum Image", and "(Un)Check All Masses" (Figure 7.2.1).

\begin{tabular}{|c|c|c|}
\hline Edit & Tools & \\
\hline y & Copy Checked Mass(es) & Ctrl + Shift + C \\
\hline 造 & Copy Sequence Table & $\mathrm{Ctrl}+\mathrm{Alt}+\mathrm{C}$ \\
\hline 政 & Copy Spectrum Image & \\
\hline & (Un)Check All Masses & $\mathrm{Ctrl}+\mathrm{A}$ \\
\hline
\end{tabular}

Figure 7.2.1 Menus for Edit option

- "Copy Checked Mass(es)" will copy selected items in the Mass List (i.e. items with a checkmark in the checkbox). The copied item can be pasted in other documents such as Notepad or Microsoft Excel for further editing.

- "Copy Sequence Table" will copy the image in the Sequence Table. This function is the same as previously shown in Section 5.2 Save Images.

- "Copy Spectrum Image" will copy the image in the Spectrum View. This function is the same as previously shown in Section 5.2 Save Images.

- "Select All" will check all the entries in the Mass List.

\subsection{View}

Items under "View" include "Results View", "MassList Toolbar", "Status Bar", "Sequence Table” (Figure 7.3.1).

\begin{tabular}{|ll|} 
View & Tools Help \\
$\checkmark$ & Results View \\
& MassList Toolbar \\
$\checkmark$ & Status Bar \\
$\checkmark$ & Sequence Table \\
\hline
\end{tabular}

Figure 7.3.1 Menus for View option

A checkmark next to each item indicates that the corresponding GUI element is visible. For detailed information regarding the location of each panel, refer to Section 2 Interface Layout. These items allow users to reopen a specific panel if it has been closed. 


\subsection{Tools}

Items under "Tools" include "Workflow Manager", "Quick Decon. Parameters", "Paste Sequence" and “Configuration” (Figure 7.4.1).

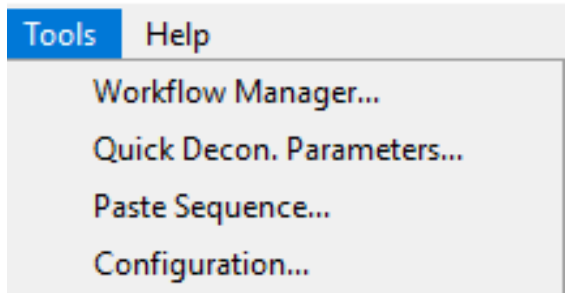

Figure 7.4.1 Menus for Tools option

- The "Workflow Manager" is a dialog that controls the data analysis workflow for top-down proteomics. This tool is activated when a workflow such as deconvolution or database search is activated. If the user closes the Workflow Manager, it can be reopened using this menu selection.

- "Quick Decon. Parameters" allows users to change parameters for the "Quick Deconvolution" function.

- "Paste Sequence" changes the sequence displayed in the Sequence Table panel. The function of the button is the same as "Modify Sequence" in the Sequence Table and "Paste Your Sequence" under "Characterization" within the Targeted Mode workflow.

○ "File Paths" tab. For more information regarding the deconvolution and database search, please refer to the User Installation Guide included in the MASH Explorer software installation regarding installation of each deconvolution and algorithm (Figure 7.4.2). Users can use "Find" option to attempt to automatically locate the relevant directory.

○ If the file is not found, the "Browse" option will permit users to manually specify the directory of the selected software.

○ Users can click the "Download" button to access the software download page for a given software app. Green indicates that the target file can be located, while red indicates that the target file is not found in the specified directory.

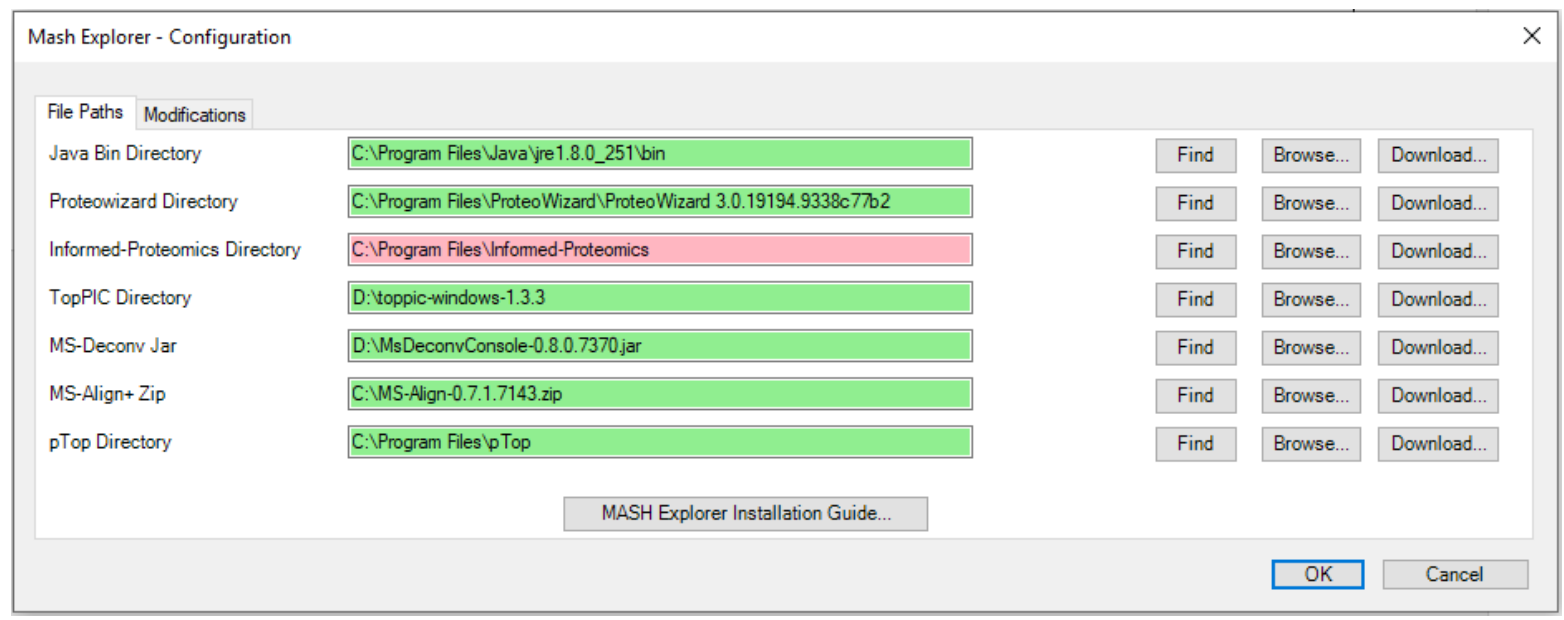

Figure 7.4.2 MASH Explorer - Configuration for software 
- "Modifications" tab. Users can also add new protein modifications or edit existing modifications (Figure 7.4.3). The name, monoisotopic mass and average mass can be input. Press "OK" to save the modifications, and the modifications will be added in the PTM list in the Sequence Table panel.

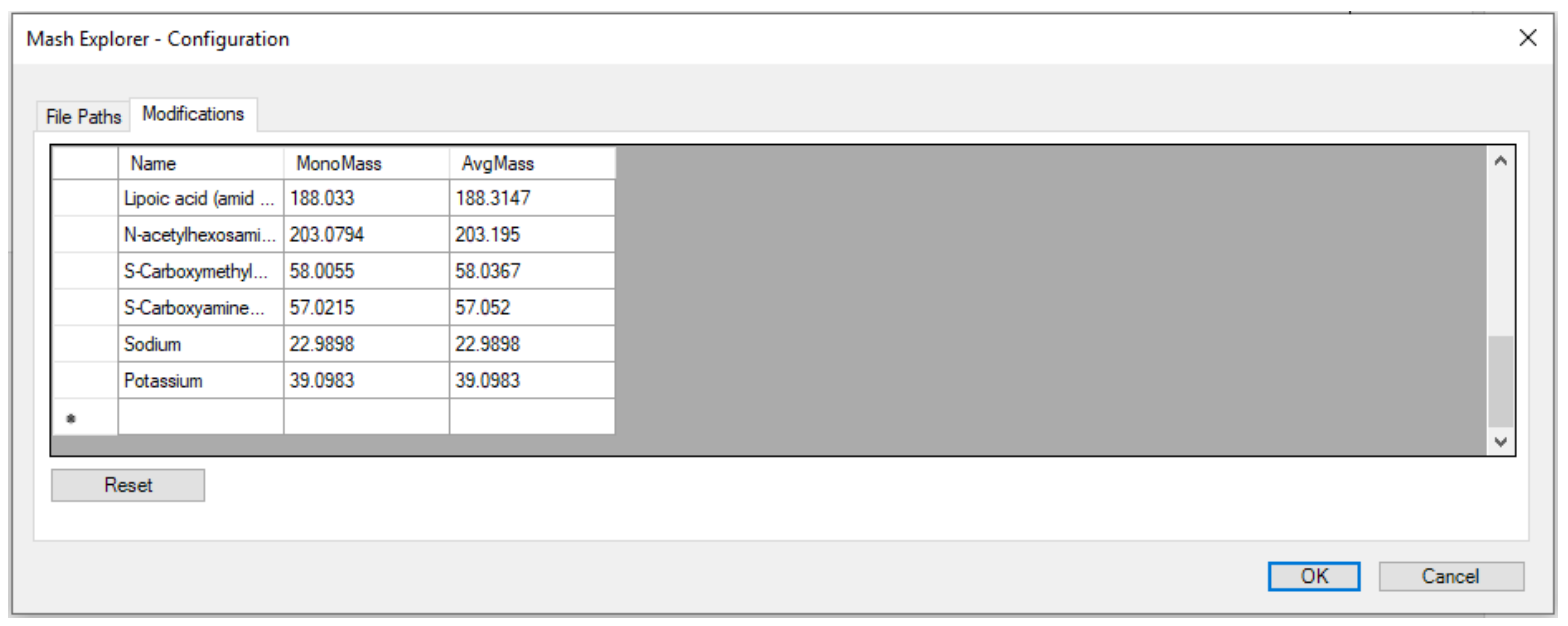

Figure 7.4.3 MASH Explorer - Configuration for modifications

\subsection{Help}

Items under "Help" include "Getting Started" and "About MASH Explorer" (Figure 7.5.1).

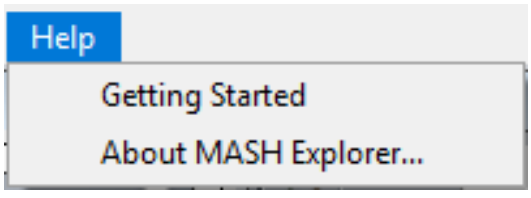

Figure 7.5.1 Menus for Tools option

- "Getting Started" will open the following website webpage:

http://labs.wisc.edu/gelab/MASH_Explorer/GettingStarted_MASHExplorer.htm, which introduces the basic functions in MASH Explorer software.

- "About MASH Explorer" provides the software information and acknowledges all the supporting algorithms.

Users can also contact MASH team support via email (mash-support@lists.wisc.edu). 


\section{Configuration and Log Files}

The MASH Explorer application uses a number of configuration files, and a log file, to store information. These files are found in the folder $<$ user $>\backslash$ AppData $\backslash$ Local $\backslash$ MASH Explorer. For example, if your user name is johndoe, your configuration and $\log$ files would be found in the folder $\mathrm{C}: \backslash$ Users $\backslash j$ ohndoe $\backslash$ AppData $\backslash$ Local $\backslash$ MASH_Explorer if your home folder is in the usual location.

\subsection{Configuration Files}

When you first start MASH Explorer, it will automatically create a number of configuration files in the MASH_Explorer folder. These configuration files are used to preserve your user preferences across runs of the software. You can change configuration values via the "Tools" -> "Configuration" menu option. The Process Wizard also allows you to change the configuration for both Quick Decon and "normal" deconvolution/search processing. (If necessary, the configuration files can also be manually edited with a tool such as Notepad; however, this should only be done in the case of faulty configuration that prevents MASH Explorer from running, because it is possible to produce an invalid configuration file if you manually edit it.)

Currently MASH Explorer uses the following configuration files:

- MASH_Explorer_FilePaths.txt

- MASH_Explorer_NormalDeconSearch.txt

- MASH_Explorer_QuickDecon.txt

- MASH_Explorer_Modifications.xml

In the future the number of configuration files will increase as we increase the flexibility of the MASH Explorer software.

\subsection{Log File}

MASH Explorer will create a log file called MASH_Explorer_LogBook.txt when it runs. Each time you run MASH Explorer, the log file will be appended to - it will never be overwritten. (If you are concerned about the log file getting too big, you can manually delete or rename it.)

The MASH Explorer log file contains a record of the actions you perform each time you run the application.

When you start MASH Explorer, the log file will contain three lines like this:

$$
\begin{aligned}
& \text { 2019-05-28 11:26:03: Log file opened/created } \\
& \text { 2019-05-28 11:26:03: MASH Explorer version 1.0.0.20579 }
\end{aligned}
$$

This tells you when MASH Explorer was started, and what the version of the software is. 
The log file also contains some information about the versions of other software packages used by MASH Explorer -- for example:

2019-05-28 11:27:00: ProteoWizard version: 3.0.19014

The log file contains information about all of the processing you do while running MASH Explorer. This can be useful if you need to go back and figure out what processing you did on a given data set.

The log file can also be useful if you run into any problems with MASH Explorer - if you need to ask us for help with a problem, please preserve the relevant log file so that you can send it to us if necessary. 